Cuadernos de Ilustración y Romanticismo

Revista Digital del Grupo de Estudios del Siglo XVIII

Universidad de Cádiz / ISSN: 2173-0687

$\mathrm{n}^{\circ} 25$ (2019)

\title{
LA POLITICA MARITTIMA DI BERNARDO TANUCCI NELL'EPISTOLARIO CON CARLO III RE DI SPAGNA
}

Maria Sirago

(NAV Lab, Laboratorio di Storia Navale)

Recibido: 06-01-2019/Revisado: 09-06-2019

Aceptado: 09-06-2019 / Publicado: 20-12-2019

Resumen: Ministro de confianza de Carlos de Borbón, después de haber ocupado cargos de prestigio hasta 1758, cuando Carlos partió para España, Tanucci fue elegido por el rey como miembro autorizado del Consejo de regencia hasta 1767, cuando Fernando IV alcanzó la mayoría de edad. En esos largos años escribió semanalmente al rey de España para informarle sobre las cuestiones del reino napolitano. El vinculo con España había conducido en parte al sacrificio del desarrollo comercial napolitano, aunque se había inciado un plan de reorganización de la marina, una cuestión debatida en la correspondencia de Tanucci. Pero en 1775, cuando la reina María Carolina se unió al Consejo de Estado (despues del nacimiento de su primier hijo, según las cláusulas de las capitulaciones matrimoniales) la política filohispánica de Tanucci ya no se toleraba. Por lo tanto, al año siguiente la reina lo invitó a retirarse a la vida privada, aunque él continuó intercambiando cartas con el rey Carlos III hasta su muerte.

Palabras Clave: Tanucci correspondencia, flota, marina mercante, desarrollo comercial.

\section{THE MARITIME POLICY BY BERNARDO TANUCCI IN THE EPISTOLARY WITH CHARLES III KING OF SPAIN}

ABSTRACT: Trusted advisor of Charles of Bourbon, after having held prestigious positions until 1758, when Charles left for Spain, Tanucci was chosen by the king as an authoritative member of the Council of Regency until 1767, when the king Ferdinand reached the age of majority. In those long years he wrote weekly to the king in Spain to brief him on the questions of southern kingdom. The political relations with Spain had a dramatic impact on Napolitan comercial development, but there were plans for the reorganization of the Royal Navy, a question debated in Tanucci's correspondence. However, in 1775, when Queen Maria Carolina joined the Council of State (after the birth of her first son, according to the clauses of the marriage chapters), Tanucci's pro-Spanish foreing policy was no longer tolerated. Therefore, the following year was invited by the queen to retire to private life, although he continued to exchange letters with the king Charles III until his death.

KeYwORDS: Tanucci's correspondence, Royal Navy, merchant navy, commercial development. 


\section{INTRODUZione}

Consigliere di fiducia di Carlo di Borbone, dopo aver ricoperto incarichi prestigiosi fino al $\mathrm{I}_{75}$, quando Carlo partì per la Spagna, fu scelto dal re come membro autorevole nel Consiglio di Reggenza fino al 1767 , quando Ferdinando IV raggiunse la maggiore età. In quei lunghi anni scrisse settimanalmente al re in Spagna per ragguagliarlo sulle questioni del regno meridionale. In effetti il legame con la Spagna ha comportato in quel periodo il sacrificio dello sviluppo commerciale napoletano anche se si è provveduto ad una riorganizzazione della marina, sia regia che mercantile. Difatti nel 1765 Tanucci scriveva a Carlo che bisognava riorganizzare il comparto marittimo con la costruzione di vascelli, sciabecchi e galere, necessari per la difesa delle coste e della marina mercantile. Nel contempo veniva riorganizzata l'Accademia di Marina per gli ufficiali da istruire per manovrare i nuovi vascelli che si andavano costruendo. Poi nel 1770 vennero create sul modello proposto da Antonio Genovesi le scuole nautiche di Napoli e Sorrento, volte a formare un ceto marinaro consapevole ed istruito, capace di effettuare viaggi in mari lontani (Baltico e Mar Nero) e nelle Americhe per incrementare il commercio (Sirago, 2019: 63 ss). Tali questioni sono state a lungo dibattute nel carteggio tanucciano con il re Carlo. Si è detto infatti che Tanucci non era interessato alla politica marittima; ma bisogna osservare che la contraddittoria politica della Reggenza derivava dallo stretto legame con quella spagnola con cui bisognava fare i conti. Questo si verificò anche dopo il $\mathrm{I} 767$, al termine della Reggenza. Ma nel $\mathrm{I775}$, quando la regina Maria Carolina entrò nel Consiglio di Stato (dopo il parto del primogenito, secondo le clausole dei capitoli matrimoniali), la politica filoispanica del Tanucci non fu più tollerata. Perciò egli l'anno seguente fu invitato dalla regina a ritirarsi a vita privata, anche se continuò a scambiare missive con Carlo fino alla morte.

\section{Il «tempo eroico» di Carlo di Borbone}

Quando Carlo di Borbone conquistò Napoli, nel I734, i ministri che lo attorniavano, in primis Bernardo Tanucci, cercarono di attuare un nuovo sistema amministrativo per far progredire la vita economica e civile del Regno. In parte si continuò a seguire la politica mercantilistica introdotta durante il viceregno austriaco (I707-I734); ma ben presto i ministri si resero conto che bisognava riorganizzare le strutture politiche ed economiche del nuovo regno, gestito da un Consiglio di Stato formato da due spagnoli, Josè di Montealegre, marchese di Salas (poi duca), segretario di Stato, e José Manuel de Benavides y Aragón, conte di Santisteban del Puerto, e da alcuni napoletani, tra cui il principe di Francavilla Michele Imperiali e il duca Gaetani di Laurenzano (Ajello, I976: 480 ss). Un componente importante del governo era il toscano Bernardo Tanucci, esperto giurista, venuto a Napoli al seguito di Carlo; qui fu nominato Consigliere di Stato, quindi ministro di Giustizia nel I75I e due anni dopo ministro degli affari esteri, poi Segretario di Stato nel I754. Egli era un fiero assertore della necessità di combattere il sistema feudale in cui il Regno era avviluppato (Tanucci, I980a: 89I), e di ridimensionare la giurisdizione ecclesiastica, entrambi causa dell'arretratezza del meridione e della Sicilia. Seguendo il suo obiettivo egli si dedicò con ogni energia alla creazione di un grande Stato nell'Italia meridionale, attraverso un riformismo politico-giuridico e istituzionale attuato con indubbia passione civile, sia pure con esiti parziali e talora discutibili (Cernigliaro, 20I2). In un primo momento la situazione economica apparve molto critica: a fine 1734 il re, a capo dell'esercito partì alla conquista della Sicilia; e ciò costò molto denaro, anche 
se arrivarono fondi dalla Spagna, per cui si dovette ricorrere a consistenti prestiti dalle banche napoletane e l'incauta promessa di condono fiscale fu annullata (Ajello, 1977).

Fin dai primi mesi di governo si sviluppò un ampio dibattito sul modo di riorganizzare il commercio e creare uno sviluppo nel nuovo regno indipendente: agli inizi del 1735 Gregorio Grimaldi nelle sue inedite Considerazioni trascritte e commentate da Renata Pilati riprese le idee espresse dal Valignani in epoca austriaca dando suggerimenti sia di tipo protezionistico, come la vendita ed il consume di panni lavorati in regno, sia di tipo liberistico, come quello di ridurre i dazi, ripristinari i porti, proporre la creazione di porti franchi, costruire lazzaretti, creare compagnie di commercio, facilitare la concessione di permessi per armare navi «in corso», insomma riorganizzare tutti i settori economici. ${ }^{1}$ Nel I735 fu istituita una «Giunta di Commercio» su modello di quella austriaca, sostituita nel I739 dal Supremo Magistrato di Commercio in cui si decideva in merito alle cause commerciali e maritime discusse nei «Consolati di terra e mare» aperti in tutti i principali porti. Ma poichè il sistema era dispendioso Tanucci il 6 agosto I746 scriveva al principe Bartolomeo Corsini che si era deciso di conservare solo quelli dei porti più importanti, Napoli, Reggio, Crotone, Gallipoli, Barletta, Manfredonia, con giurisdizione «ristretta alle sole cause del commercio esteriore, fra i forestieri o tra forestiero e paesano» (Tanucci, I980b: 90-92). Gli altri furono accorpati nelle preesistenti Deputazioni della Salute che controllavano l'avvenuta quarantena delle merci provenienti da paesi in qui vi era il sospetto di peste. Infine lo stesso I739, il Io giugno, si tenne la prima riunione della «Conferenza di commercio», un nuovo organo che si doveva riunire settimanalmente nella Segreteria di Stato, Guerrra e Marina per discutere su questioni di commercio, ripresa degli scambi commerciali, manifatture, diritti fiscali; uno dei primi compiti era però quello della riorganizzazione dei principali porti per i quali fu create una Soprintendenza dei Porti e Moli del Regno che doveva redigere i progetti affidati alla singole Giunte, soggette al controllo della Segreteria di Guerra, sotto la supervisione dell'ingegnere di origini torinesi Giovanni Bompiede, capitano del porto di Napoli (Pezone, 2006) e di Michere Reggio, generale della flotta, a capo della Giunta di Marina (Luise, 20I6), che dovevano organizzare il lavoro nei singoli porti, in primis quelli della Capitale (Sirago, 2004: 34).

Negli anni seguenti fu intrapresa l'opera di ricostruzione del Regno, il che comportò un forte impegno finanziario. In primis fu posta in cantiere la flotta, da ricostruire, visto che galere e vascelli costruiti dagli Austriaci erano stati portati a Trieste o distrutti (Sirago, 2016). L'armamento era necessario per la lotta alla dilagante pirateria, che compiva attacchi alle coste napoletane e sicule, e per la protezione delle navi mercantili. Furono poi intraprese grandi opere pubbliche, in particolare il ripristino dei porti più importanti, a partire da quello quello della Capitale, dei lazzaretti, dell'arsenale, (Sirago, 2004: 33 ss; Marin, 20I7). Inoltre si provide a ripristinare le fortificazioni di tutto il Regno (Sirago, 2008).

Uno dei problemi più urgenti da risolvere era quello del commercio estero, poco sviluppato a causa dei secolari attacchi alle navi napoletane e siciliane dei continui attacchi turchi e barbareschi, in primis quelli di Algeri (Wolf, 1979) e Tunisi, formatisi nell'Africa settentrionale sotto la protezione dell'Impero ottomano (Bono, 2008). Questa situazione era rimasta inalterata per tutto il periodo della dominazione spagnola e austriaca in Italia meridionale al punto che per poter commerciare le navi mercantili dovevano essere protette dalle flotte di galere e vascelli da guerra costruite in epoca spagnola (Sirago, 2018a) e austriaca (Sirago, 2017). Ma già in epoca austriaca le relazioni

I Biblioteca Corsiniana, Roma, Cod. Cors. $1348=$ ms. 35 C 27, inizi del I735, Gregorio Grimaldi, Considerazioni intorno al commercio del Regno di Napoli. 
economiche tra Ottomani e Napoletani furono regolate da un trattato di commercio e navigazione, considerate da Federico Valignani il miglior strumento per incoraggiare gli scambi commerciali (De Tiberiis, 200I; Mafrici, 20I2a). Il 2r luglio I7I8, alla fine della «Seconda Guerra di Morea» (Setton, 199I), l'imperatore d'Austria Carlo VI firmò la pace di Passarovitz in cui si garantiva libero commercio tra la Porta e l'Austria anche nel Mar Nero (Bianchini, 197I): questo trattato fu poi il modello dei trattati con gli Stati Barbareschi (Schipa, 1923). Pochi giorni dopo la firma del trattato di pace, il 27 luglio, venne firmato con la Porta un trattato di commercio, che servì a contenere gli assalti dei corsari nemici (Sirago, 2or6: 8-9). Secondo il parere di Ludovico Bianchini «l'unico trattato che apportò qualche vantaggio fu quello del $\mathrm{I}_{225}[\ldots]$, inoltre due convenzioni del I726 fatte con gli Stati d'Algeri e Tripoli che [permisero ai] nostri concittadini [di potere] colà liberamente trafficare; ma poiché inutile era venire a nudi patti con quei pirati, così niun bene a noi ne derivò, e al contrario le marine del nostro reame seguitarono ad essere esposte alle loro incursioni» (Bianchini, 1971: 340).

Pochi anni dopo la conquista del Regno i ministri decisero di riprendere la politica austriaca per poter riorganizzare il commercio in Levante, dove il monopolio era detenuto dalla città di Marsiglia, dichiarata porto franco nel I669, che commerciava con i vari scali (Carrino, 2017). Si continuò ad osservare il privilegio di porto franco per Messina, concesso nel 1695, riconfermato nel I728 dagli austriaci, poi da Carlo di Borbone e nel I785 da re Ferdinando (Bottari, 20I8: $80 \mathrm{ss}$ ). Si decise perciò di intavolare trattative commerciali con la Porta e gli stati barbareschi per incrementare il commercio in Levante: il 7 aprile I740 fu firmato un trattato di commercio con la Porta, in modo da creare condizioni favorevoli agli scambi commerciali. Il trattato fu poi la base di quello firmato il 3 giugno I74I tra Napoli e la Reggenza di Tripoli (Pirolo, 20I7), un centro molto attivo, anche se di livello inferiore a Costantinopoli (Bono, 2016). Un ricordo di questo memorabile evento è raffigurato in due dipinti conservati nella sala del trono del Palazzo Reale di Napoli commissionati da Carlo al pittore di corte Giuseppe Bonito: il primo rappresenta Haci Hiiseyin Efendi, inviato speciale del sultano ottoman Mahmud I (I730-I754) alla corte di Napoli (Musella Guida, 2or6). Il secondo è il ritratto di Mustafa Bey di Derna, inviato speciale di Ahmed Karamanh, bay di Tripoli, con la sua scorta (D'Amora, 2003).

In realtà il commercio col Levante non era decollato dopo il trattato del I740, come si era sperato; ma Annastella Carrino dai dati raccolti evince l'esistenza di una sorta di «commercio obliquo»: difatti i genovesi, per aggirare il blocco derivante dai nuovi divieti e restrizioni imposti da Marsiglia, trasportavano una buona parte del traffico col Levante sulle coste meridionali, fra i piccoli approdi calabresi e il porto franco di Messina, da dove le merci, che ormai risultavano regnicole, ripartivano per Marsiglia (Carrino, 20I7: IoI ss).

Per riorganizzare le strutture commerciali negli anni '40 Carlo, nell'ambito della ristrutturazione del fronte a mare tra il molo grande e il porto commerciale del Mandracchio, vicino al Carmine, diede ordine a Domenico Antonio Vaccaro di costruire all'Immacolatella Vecchia un palazzo per la sede della Deputazione della Salute (Pessolano, 1993). In questa sede dovevano essere conservate tutte le merci in arrivo, specie quelle provenienti dal Levante, che dovevano rimanere in quarantena a «purgarsi». Invece le navi dovevano approdare nelle vicina isoletta di Nisida dove era stato costruito il lazzaretto (De Rinaldi, 2004) in cui si dovevano trascorrere i quaranta giorni prescritti dalla legislazione sanitaria vigente emanate per prevenire i contagi di peste (Bergoldt, 2004).

Pochi anni dopo la firma dei trattati Bernardo Tanucci scriveva al marchese di Salas: «Lindustria dell'uomo e del governo si suol dirigere a questo fine, principalmentte collo stabilire il commercio». Egli sottolineava che i veneziani erano abituati a commerciare 
con gli infedeli; perciò per poter incrementare i commerci bisognava confermare la pace coi tunisini anche se essi chiedevano 38.000 zecchini veneziani e auspicava di fare la pace anche con gli algerini e i tripolini, altrimenti la navigazione non sarebbe mai stata sicura. Ma Francesco Ventura, presidente del Supremo Magistrato di Commercio, manifestava i suoi dubbi: in una lettera a Tanucci del 30 gennaio 1743 egli scriveva che se si dava una somma ai tunisini, gli algerini avrebbero voluto il doppio, anche se era inevitabile concludere il trattato con Tunisi per decoro del re e del popolo, altrimenti si sarebbe scatenata la rabbia «di quei barbari» (Tanucci, I980a: 659-664). In realtà l'ago della bilancia fu sempre la Porta Ottomana, dove nel 1747 era stato nominato come console napoletano Guglielmo Ludolf: ma egli non riuscì a far sviluppare proficui scambi commerciali con Napoli, anchè perche il regno, privo di manifatture, non poteva offrire merci appetibili per la nobiltà turca, come quelle veneziane e francesi. Il ruolo di Ludolf fu però di primaria importanza per dirimere gli attriti con gli stati barbareschi, pur sempre nell'orbita del governo turco (Mafrici, 2004). Lo stesso Tanucci in una lettera al Ludolf del 28 agosto I756 notava come fosse difficile commerciare con Costantinopoli, dove le merci napoletane erano meno richieste di quelle francesi, più raffinate, concludendo: «Forse verrà un giorno in cui il commercio che costì faccia la nostra Nazione le dia la consolazione alla quale anela», ribadendo lo stesso concetto il 25 dicembre (Tanucci, I984: I50 e 33I).

Perciò i rapporti epistolari tra i due ministri sono una fonte preziosa per ricostruire le vicende del commercio col Levante, che si possono evincere anche dai documenti delle legazioni consolari conservati nell'Archivio di Stato di Napoli nella sezione degli Affari Esteri (Rao, 20I7).

I trattati con la Porta e con Tripoli erano stati stipulati per incrementare il commercio col Levante (Clemente, 20I7) e limitare gli assalti barbareschi (Siking e Sola, 20I4). Ma Carlo voleva far sviluppare anche il commercio con i mari del Nord: da lì proveniva gran quantità di pesce salato, necessario per i periodi di magro prescritti dal calendario liturgico, per l'approvvigionamento della Capitale, circa 40.00o cantara verso il 1770 (Pagano De Divitiis e Giura, 1997: 106). Inoltre erano necessari alcuni materiali per la flotta, alberi del Nord, da usare per gli alberi dei nuovi vascelli (invece di quelli di Calabria, meno affidabili e quasi esauriti), cannoni di bronzo, ecc. (Sirago, 2orza). Perciò sulla scia dei precedenti trattati nel $\mathrm{I} 742$ fu stipulato un trattato di commercio con la Svezia, nel I748 con la Danimarca e nel I753 con l'Olanda (Galanti, I968: I, I86). Poi l'abate Ferdinando Galiani, che si era occupato delle trattative dei precedenti trattati commerciali insieme ad Antonio Genovesi, cercò di stipulare un simile trattato con la Francia: ma il tentativo non andò a buon fine, in quanto la Francia, forte del suo consolidato commercio con il Regno, specie attraverso i porti di Marsiglia e Nizza, non riteneva necessario un simile trattato. Perciò Bernardo Tanucci per controllare l'operato dei francesi agli inizi della Reggenza, il I5 marzo I76o, nominò l'abate Galiani incaricato d'affari a Parigi presso l'ambasciatore napoletano, il conte di Cantillana, Giuseppe Baeza y Vicentelo (Diaz, r968).

Per incrementare i commerci in Mediterraneo fu data particolare attenzione alla ricostruzione della flotta, necessaria per la difesa della marina mercantile: dal 1736 erano state armate per ordine del re due grandi galeotte, imbarcazioni ad un albero con tre cannoni e alcune petriere ed erano stati promessi sussidi per coloro che avessero armato simili imbarcazioni, invito raccolto solo nel 1749 da alcuni commercianti di Napoli e Castellammare (Sirago, 2004: 36). Poi vennero costruiti altri scafi per la flotta: ma si decise di creare una marina agevole atta alla difesa e alla corsa. Perciò furono costruite poche grosse unità. Alla partenza di Carlo per la Spagna si contavano il vascello San Filippo la Reale, a 60 cannoni, varato nel 1737 , e tre fregate da 40 cannoni, Regina, Concezione e Santa Amalia. Il resto era costituito da agevoli imbarcazioni adatte alla 
guerra di corsa, 4 galere Capitana, Patrona, Sant'Antonio e San Gennaro, alcune galeotte e la squadra di sei sciabecchi da 20 cannoni, S. Gennaro, S. Pasquale, S. Ferdinando, S. Gabriele, S. Luigi, S. Antonio (Mafrici, 2007: 647). Per organizzare le costruzioni navali in quel periodo erano stati mandati uomini in Inghilterra e Olanda per apprendere le nuove tecniche costruttive (Salzano, I924: $37 \mathrm{ss).} \mathrm{Si} \mathrm{costruivano} \mathrm{vascelli} \mathrm{e} \mathrm{fregate} \mathrm{e} \mathrm{dal} \mathrm{I} 749$ le galere vennero lentamente sostituite dagli sciabecchi costruiti su modello spagnolo, velieri mediterranei di origine araba con scafo con prua slanciata, a tre alberi, talvolta con un piccolo bompesso, molto manovrabili e veloci (Formicola-Romano, I990). Tale svolta nella tecnologia nautica si evince anche dalla netta diminuzione dei remieri, spesso schiavi musulmani, sostituiti dalla propulsione velica (Varriale, 20I7).

In quel periodo si decise anche di regolamentare la navigazione per le navi mercantili con una prima prammatica del I74I in cui si prescriveva che le imbarcazioni dovevano ottenere un permesso, da rinnovare ogni due anni, per i viaggi che intendevano intraprendere. L'anno seguente fu emanato un Regolamento, rinnovato nel I75I, I757 e I759, e fu creato un comitato che doveva controllare loperato dei capitani, obbligati a loro volta a redigere un giornale di bordo. Infine nel I75I fu creata una «Compagnia di assicurazioni maritime» per regolamentare il commercio. Vennero poi concesse franchigie per la costruzione di navi mercantili, il che dette i suoi frutti: tra il il 1572 ed il I 762 vennero costruite 444 imbarcazioni in particolare a Piano e Meta di Sorrento, a Castellammare e nell'isola di Procida, dove esisteva una antica tradizione cantieristica (Sirago, 2004, 33 ss; Passaro, 2019).

Il commercio come in passato continuò ad essere controllato dagli stranieri, anche se durante la «Guerra dei Sette Anni» ( $1757^{-1} 763$ ) si comincio ad organizzare il commercio con le Americhe. Il mercante e armatore messinese Gaspare Marchetti, che risiedeva a Londra, aveva costituito una società con alcuni mercanti, tra cui il leccese Lucio La Marra ed alcuni commercianti napoletani, Nicola Palomba e Gennaro Rossi, interessati al «commercio con l'Oceano». Così il Marchetti aveva acquistato una nave, La Concezione, che nel 1756 aveva caricato merci alla fiera di Salerno. Poi aveva ordinato al La Marra di far costruire alcune navi da 20022 cannoni per le quali aveva ricevuto le franchigie prescritte, tra cui l'acquisto di cannoni e polvere al prezzo pagato dalla regia marina. In breve tra Castellammare e Sorrento furono costruite tre navi, il Principe Reale, la Principessa Reale e la Partenope, armate dei necessari cannoni acquistati dai mercanti Palomba e Rossi. Così iniziò un proficuo commercio con la Martinica francse (Sirago, 2004: 38). Tanucci in una lettera scritta da Pisa al Ludolf del 20 aprile 1757 ribadiva che era necessario ampliare la rete dei lazzaretti: egli notava «con un poco di forza marittima, che va formandosi, e con un poco di perfezione, che va acquistandosi nelle fabbriche delle sete, ci vedremo più vicini alla speranza del commercio orientale», concludendo con rammarico che «Sarebbe stata questa guerra tra Francia e Inghilterra [la Guerra dei Sette Anni] una buona occasione, se fossimo stati più preparati» (Tanucci, 1984: 523). Difatti erano pochi i commercianti intraprendenti come il Marchetti ad aver osato solcare gli Oceani, dove vi era il pericolo del sequestro delle navi da parte degli inglesi.

Ma un nuovo corso si apriva per il regno meridionale: il 6 ottobre 1758 Carlo di Borbone, divenuto re di Spagna, cedette al terzogenito Ferdinando gli «Stati e Dominj italiani» (regni di Napoli e Sicilia, Stato dei Presidi di Toscana, beni allodiali, o personali, farnesiani e medicei) dove aveva regnato per 25 anni istituendo un Consiglio di Reggenza per amministrare i regni durante la minore età del giovane re: e designò come Presidente della Reggenza proprio il fedele segretario di Stato, Bernardo Tanucci, che da quel momento cominciò a scrivere una missiva settimanale a Carlo per informarlo delle 
questioni di governo, ufficio che continuò ad esercitare anche dopo la sua destituzione (I776), fino alla sua morte (Borreca, I976).

\section{La reggenza di Bernardo Tanucci (I758-i 767 )}

Nell'ottobre del ${ }_{1758}$ il re Carlo, stilò delle puntuali «Istruzioni [...] emesse nel lasciare Napoli», istituendo il Consiglio di Reggenza, che doveva riunirsi tutti i lunedì: particolare attenzione fu data alla Segreteria di Guerra che «doveva aver gran cura delle tre Accademie, dell'Artiglieria e degl'Ingegneri e delle Guardie Marine» (fondata nel I735); inoltre «tutti gli affari della Marina attinenti al Militare [dovevano] essere diretti dal Capitan Generale [Michele Reggio] e gli economici per l'intendente della Tesoreria Generale».2

Tanucci fin dall'inizio della Reggenza dové affrontare un difficile periodo di transizione attenendosi a tutte le indicazioni sulla politica estera che Carlo inviava settimanalmente nella sua corrispondenza ed inviando a sua volta sia al re che ai ministri della corte madrilena tutte le informazioni sul Regno (Anes, 1976). Il suo ruolo in seno al Consiglio fu di primaria importanza, data la sua personalità: ovviamente il re lo aveva scelto per le sue indubbie capacità politiche, ma anche perché in lui riponeva assoluta fiducia. Perciò il ministro enunciò un vero e proprio programma politico, «mantenere il sistema e il pensiero paterno», sia pure con prudenza, per non urtare la suscettibilità degli altri componenti del Consiglio (Maiorini, I985: XxIII-Xxiv).

Uno dei primi problemi era l'inquietudine suscitata dalla guerra tra Francia e Inghilterra, in cui furono coinvolte le principali potenze dell'epoca, che creava difficoltà per il commercio, già ostacolato dagli assalti barbareschi (Mafrici, 2007: 647 ss.), che ebbe termine solo nel I763 (Füssel, 2013). Franco Venturi ha richiamato l'attenzione sugli anni '6o, un periodo di transizione tra i vari progetti formulati e la realizzazione delle grandi riforme, quasi una "primavera dell'Illuminismo» (Venturi, I968: xvi). Nel quadro internazionale dominava la guerra che logorava l'Europa i cui effetti si riverberavano anche sui paesi non coinvolti direttamente, come il Regno meridionale, il che era ben presente nell'epistolario di Tanucci (Maiorini, 1985: x).

Il ministro aveva la piena consapevolezza della crisi attraversata dal Regno governato da un «re pupillo», sia pure sotto la supervisione della Spagna. Perciò manifestava tutta la sua preoccupazione nell'ambito della difesa, affidata ad una marina poco funzionale, malgrado tutti i ducati spesi negli anni precedenti. La marina era necessaria per lo sviluppo del commercio ma mancavano i fondi per le costruzioni: il i7 gennaio i76o l'Eletto del Popolo aveva chiesto di allestire due sciabecchi per l'inizio di febbraio per scortare i bastimenti mercantili diretti in Levante per caricare grano. Ma la richiesta non era stata accolta perché gli sciabecchi non erano ancora stati sistemati: si concesse però che essi scortassero le navi al ritorno col carico. ${ }^{3}$ Tanucci continuava a dare ordini per tenere la marina in efficienza, vendendo a Malta due piccoli sciabecchi inutili $i^{4}$ e facendo continuare le costruzioni, per le quali fu ordinato di tagliare in Sila 50 alberi. ${ }^{5}$ Inoltre il ministro chiedeva informazione ai costruttori in merito al numero e alle dimensioni degli alberi che si pensava di acquistare al Nord, in Svezia, con l'intermediazione della Corte

2 Archivio di Stato, Napoli, d'ora in poi ASN, Casa Reale Antica, 856, «Istruzioni di Carlo III emesse nel lasciare Napoli», con istituzione del Consiglio di Reggenza, s. d. ma ottobre $175^{8}$

3 ASN, Farnesiano, 1517, f. 187, I7/I/1760.

4 ASN, Farnesiano, I518, ff. 40t.-4I, 25/10/17759.

${ }_{5}$ ASN, Farnesiano, I519, f. 205, 3/7/1760. 
di Spagna. ${ }^{6}$ Nel contempo gli sciabecchi continuavano a praticare il corso con le galere e galeotte «nelle coste del Mezzogiorno della Sicilia» e in quelle di Calabria, a difesa delle navi mercantili. ${ }^{7}$

Alcuni capitani continuarono a commerciare con l'Inghilterra, approfittando del perdurare della guerra, che limitava i movimenti delle navi inglesi. Vennero infatti presentate le consuete richieste di 2 cantara di polvere «al prezzo della Corte» per armare le navi cariche per il «commercio [...] nell'Oceano» dai capitani Giosuè Cafiero e Carlo Gattola. ${ }^{8}$ Furono concesse anche diminuzioni di diritti doganali, come per il passato, per i negozianti che facevano il commercio di importazione ed esportazione con l'Inghilterra»: a titolo di esempio il 29 novembre 1759 fu accordato al negoziante don Gennaro Buono «il rilascio del $5^{\circ}$ dei diritti di dogana su merci provenienti da Londra e su quelle esportate in Inghilterra». ${ }^{9}$ Questi viaggi dei primi «capitani coraggiosi» che solcavano gli oceani erano utili non solo dal punto di vista economico ma anche da quello della diffusione dell'istruzione nautica, ancora agli albori. Il primo novembre 1759 era stato concesso a Emanuele Farina, "piloto apprendista» nel Conservatorio di Loreto, una scuola nautica istituita per una decina di «pilotini» o «grumetti» dal re Carlo dieci anni prima, ${ }^{\text {Io }}$ di poter fare un viaggio a Londra "per istruirsi nella navigazione», continuando a percepire la sua paga di «Grumetto»; ed il 24 maggio 1760 era stato concesso al pilotino Nicola Battimiello di poter compiere un viaggio a Londra sulla nave dei negozianti napoletani Nicola Palomba e Gennaro Rossi, conservando la sua paga. ${ }^{\mathrm{II}} \mathrm{Il}$ commercio, considerato «vivo fonte della felicità umana» da Nicola Fortunato nelle sue Riflessioni dedicate alla Reggenza, era uno dei capisaldi nelle discussioni in seno alla Segreteria d'Azienda (Fortunato, I760: v). Lo stesso Tanucci proponeva al Consiglio un piano per limitare i danni al commercio e alla bilancia statale derivanti dagli acquisti di merci straniere richieste per soddisfare gli aristocratici che volevano esibire a corte tutte le novità alla moda. Seguendo le idee di Antonio Genovesi (Galasso, 20I8: 60) propose di incrementare le manifatture del Regno, specie di drappi, necessari per i vestiti del sovrano e della sua corte, progettò di impiantare a Caserta, dove si stava costruendo la reggia, una «colonia di artefici» (la futura San Leucio), chiamò artefici in Regno, non permise ai "corallari» (pescatori di corallo) di Torre del Greco di trasferirsi in Toscana: mostrava di avere una spiccata visione mercantilistica volta a sviluppare attività che potessero sopperire sia ai bisogni della numerosa popolazione della Capitale che alle esigenze degli aristocratici e magari anche incrementare il commercio estero (Maiorini, I985: Ix). Ma restava il problema della difesa delle navi mercantili, che dovevano essere protette da una valida flotta.

Dopo circa un anno dalla partenza di Carlo nella lettera del 23 settembre i76o Tanucci tracciava un primo bilancio sulla situazione della flotta, riferendo con sconcerto al re: «In cattivo stato è la Marina del Regno, nonostante i 600.000 ducati annui che si spendono per essa [...] ho detto qualche parola in consiglio. Don Michele [Reggio] prende collera, e ultimamente mi disse, che li nostri mali sono universali» (Mincuzzi, I969: 5I). Egli manifestava una certa diffidenza nei confronti del generale della flotta, che a suo parere faceva spese a volte inopportune, quasi a voler favorire i suoi protetti. E spesso questo

6 ASN, Farnesiano, I518, f. 134t., 22/3/1760.

7 ASN, Farnesiano, 1519, f. 54, 17/5/1760.

8 ASN, Farnesiano, I516, f. I06, I5/II/1758, Cafiero, e I517, f. 20, II/10/1759, Gattola.

9 ASN, Farnesiano, 1517, f. 15, 29/11/1759.

Io ASN, Sezione Militare, Espedienti di Marina, 200, Io/II/I78I.

II ASN, Farnesiano, I516, f. 66, I/II/1759 e I519, f. 79, 24/5/176o. 
creava attriti durante le sedute del Consiglio di Reggenza, di cui anche il generale era membro.

Un mese dopo, il 28 ottobre, Tanucci riprendeva il discorso sulla necessità di una buona difesa. Raccontava al re che John Gray, ministro straordinario d'Inghilterra a Napoli, si era lamentato con lui perché il comandante della squadra inglese voleva entrare in Messina con 5 navi da guerra; ma il governatore di Messina principe di Villafranca, don Domenico Alliata e Giovanni, gli aveva risposto con arroganza che vi potevano entrare non più di 4 navi da guerra. Il ministro, preoccupato della massiccia presenza inglese in Mediterraneo, osservava con apprensione: «Credo che a tanto mare e a si poca forza marittima delle Sicilie, e ad una minorità non convenga, forse neppure conviene quel che talora ho pensato, cioè far noi il processo d'una preda ingiusta, e di un territorio violato, condannare il predatore per poi, se capita nei porti del Re, o eseguir la sentenza, o transigere e concordare». Infine riprendeva il discorso sulla flotta da riorganizzare, riferendo quanto detto in Consiglio: «Il capitan generale Michele Reggio ha fatto in Consiglio un discorso patetico sulla poco buona fabbrica degli armamenti di mare del Re, essendogli osservato che per poco che ciascuno di essi navighi, eccettuati gli sciabecchi, qualche parte che li scompone, li arresta nei porti. Ha esagerato la poca cura nelle carene, e acconci». Ma egli, sempre sospettoso nei confronti del Reggio, aveva ordinato di non «dare partito a' mastri d'ascia, calafati, e altri artefici [per]li acconci, ma [di farli] per tempo, e con assistenza dei rispettivi comandanti», in modo da limitare le spese inutili (Tanucci, I985: 47-54). Il I8 novembre scrisse a Stefano Reggio e Gravina, principe di Iaci, ambasciatore a Madrid (Zapperi, I96o), una lettera in merito alle costruzioni navali: in primo luogo manifestava tutto il suo dispiacere per «la perdita del vascello San Fernando che andò a traverso nella spiaggia di Cartagena per non aver le arcate tenuto, [concludendo] [...] Non abbiamo marina superflua e questa è la nostra maggiore importanza. Non so come si stia costì a costruttori di navi da guerra. Noi qui abbiamo gran necessità di uno almeno, che fosse di una perizia sicura e conosciuta» (Tanucci, I985: I28-I30). Il 30 dicembre scrisse di nuovo a Iaci un'altra lunga lettere in cui si lamentava della poca potenza marittima del Regno: in effetti questi riteneva che la spesa era infinita «e assai vano l'esistente». Ma Tanucci ribadiva:

Sempre più confermo e persisto nell'opinione di che potenze marittime devono essere e la Spagna e le Sicilie. Per quanto abbiano truppe di terra, frontiera non si può guardare, perché sul mare è immensa. Con 600.00o ducati tenghiamo quattro fregate (non contando che per fregata l'unico vascello da guerra e che a fregata di 50 e 40 pezzi si ridurrà) quattro galere, quattro galeotte e sei sciabecchi. Vede Vostra Eccellenza che non aumentando le galere con altrettanto, cioè con altri 600.000 ducati si potranno avere altre Io fregate, otto sciabecchi e otto galeotte. Il commercio protetto si aumenterebbe, questo genere di forza si potrebbe avere nello stato nel quale son più di 60 mila anime che vivono del mare e si avrebbe la frontiera portatile. Questo aumento, avuto riguardo alla spesa che si fa oggi nella truppa, non la diminuirebbe nobilmente e sempre si potrebbero avere 30 battaglioni e dieci squadroni. Mi pare che Ensenada co' suoi costruttori inglesi guastò la marina. Viva il re che ha rimessa in piedi la fabbrica dell'Avana [dove si costruiscono] vascelli [...] benissimo fabbricati, agili e forti. Questo comandante dell'Atlante [Vincente Gonzales] mi ha detto molti errori inglesi nella fabbrica di questo suo vascello. Faccia Vostra Eccellenza quanto può per trovare un costruttore (Tanucci, 1985: $250-252)$. 
Pochi giorni dopo, il 6 gennaio I76r, scriveva anche al marchese di Squillace, Leopoldo de Gregorio e di Valsantoro, segretario d'azienda di Spagna, per esprimere il suo disappunto: «L'assentista della marina si è trovato mancante in tutti li generi della sua incombenza, ed è stato con tali mancanze cagione del poco servizio che si è potuto ricevere dagli armamenti del re, li quali sono tanto necessari in questi tempi per poter sostenere il poco commercio che abbiamo. Martinez [Giuseppe, detto capitan Peppe] ha fatto un dettaglio di quel che è necessario fare, sarà tutto regolato l'anno avvenire» (Tanucci, I985: 279-280).

Secondo la relazione di Carlo Antonio Broggia del I764 si contava ancora un certo numero di imbarcazioni, un vascello da guerra a 64 cannoni, due fregate da 36 e 40 cannoni, sei sciabecchi da I4 a I8 cannoni, 4 galere e 4 galeotte o mezze galere. Ma il commercio era sempre nelle mani degli stranieri, anche egli riferiva che si contavano circa 400-500 legni mercantili, tutti costruiti nei territori compresi tra Castellammare a Vietri, nell'isola di Procida ed a Gaeta (Broggia, 1979). Il dato è confermato da quelli conservati presso l'Archivio di Stato per le costruzioni tra il $175^{2}$ ed il I762: in quel decennio furono costruite 444 imbarcazioni, soprattutto a Piano e Meta, nella costa sorrentina, e a Procida con un incremento tra il I760 ed il I76I (Sirago, 2004: 86).

In quegli anni i mercanti organizzavano viaggi in Levante, come quello di Nicola Palomba, che chiedeva la patente e la bandiera napoletana per due navi noleggiate per caricare in Levante grano destinato alla Capitale..$^{\text {I2 }}$ Anche il capitano Scarpati chiedeva una patente «per garantirsi dai Corsari barbareschi». Perciò si dava ordine al Ludolf di provvedere, anche perché lo Scarpati avrebbe pagato il prezzo dovuto. ${ }^{13} \mathrm{Ma}$ queste precauzioni non bastavano: allo stesso Ludolf si chiedeva infatti di usare «tutto il suo zelo» per ottenere dalla Porta la restituzione di una tartana napoletana di padron Salvatore Cafiero predata da corsari di Dulcigno (oggi Ulcinj) (Sbutega, 2orr: 45) presso la fortezza di Navarino, territorio turco e di un bastimento di padron Arpante da un corsaro inglese, sempre in territorio turco, già portato a Malta, richiesta rinnovata varie volte..$^{14}$ Altri reclami furono presentati per la tartana di padron Guida predata nelle acque di Candia (oggi Creta) da un bastimento tripolino: perciò Ludolf consigliava di nominare consoli regnicoli in quei porti in modo da controllare gli eventuali assalti, ${ }^{15}$ che continuavano indisturbati. L'ir dicembre I76o furono nominati consoli nel porto della Canea di Candia, ad Atene, a Napoli di Romania, a Rodi, ad Aleppo, a Durazzo, a Patrasso, a Cefalonia e viceconsoli a Spinalonga e Negroponte. Per Rodi fu nominato Nicola Maddalena, per Patrasso il signor Mainenti, per Durazzo Michele Panno e per Cefalonia don Francesco Salmon; per gli altri porti si chiese allo stesso Ludolf di suggerire i nomi di persone idonee $^{16}$

Nel contempo il Della Marra continuava ad organizzare viaggi in Oceano: il Fortunato ricorda con soddisfazione:

Già grazie a Dio si sentono velicare i nostri legni per l'America. Una Nave comandata da Capitan Michele di Costanzo Napolitano, preso il suo carico di vino, di manna ed altre mercanzie in Pozzuolo, si sono queste spacciate nella Martinica

I2 ASN, Farnesiano, 1517, ff. I2 ss., 29/11/1759.

I3 ASN, Farnesiano, I520, ff. I4 ss., I5/8/1760.

I4 ASN, Farnesiano, I520, ff. 32 ss., 2 I/ $/ 8 /$ I7 60 e ff. 250 ss., 6/II/I760, per il Cafiero e ASN, Farnesiano, I520, ff. 32 ss., 2I/8/1760, ff. 5I ss., 28/8/1760 e ff. I90 ss., I6/Io/I760, ff. 28Iss., 20/II/I76o, per padron Arpante.

I5 ASN, Farnesiano, I520, ff. I90 ss., I6/10/1760.

I6 ASN, Farnesiano, I52I, ff. 48 ss., II/I2/I760; I524; ff. II7t ss., 20/8/I76I; I523, f. 274, II/2/1762; I525, ff. II4 ss., I5/6/1762. 
[nelle Antille, possesso dei Francesi] per interesse e con interesse di don Luzio alias Luccio La Marra pubblico Negoziante di questa Piazza, con introito di un vantaggio strepitoso, avendo raddoppiato il suo Capitale col favore di tante derrate Americane. Ecco i dolci frutti d'un negozio ben regolato. Ed assicurato egli da sì considerabile vantaggio ha già spedito altra Nave di maggior portata col carico di simili generi comandato dal Capitan Stinchi di Sorrento (Fortunato, I76o: LIV).

Il La Marra aveva ottenuto «il solito quinto» di esenzione sui diritti di dogana per il viaggio che stava preparando per tornare «nelle isole francesi d'America» [Martinica ${ }^{17}$ e 6 «cantara» di polvere al prezzo della Regia Corte «per grazia speciale»; di solito se ne concedevano due, come si fece per Vincenzo di Palma. ${ }^{18}$ Ma non sempre l'esito era fortunato: nel I76r una nave del La Marra, la Vittoria, fu predata dal corsaro inglese Tohmas Reyd, per cui egli decise di andare a Londra per chiederne la restituzione. La situazione era complicata perché il La Marra aveva in corso un processo col suo socio, Gaspare Marchetti. Questi aveva creato col fratello Nicola una compagnia di commercio con vaie sedi tra cui una in Londra. Il La Marra, che vantava crediti da Nicola, aveva fatto sequestrare una nave di Gaspare in partenza per Londra; perciò il mercante aveva dovuto pagare la cauzione per poter fare partire la nave. Ma il mercante, arrivato a Londra per chiedere la restituzione della nave, era stato arrestato su richiesta di Nicola dal tribunale dell'ammiragliato britannico per insolvenza, per cui aveva dovuto pagare un'altra cauzione. Intanto a Napoli il 5 febbraio 1762 si ordinava di spedire i conti nella questione tra i Marchetti e il La Marra per esaminarli e dirimere la causa nei tribunali napoletani. ${ }^{\text {19 }}$

Il Tanucci il 2I ed il 28 luglio aveva scritto all'inviato straordinario a Londra, Giovan Battista Albertini, principe di Cimitile, di adoperarsi con ogni mezzo per perorare la causa del La Marra; ma l'Albertini, poco fiducioso nelle possibilità del giovane regno borbonico nell'ambito della politica estera, si mostrava scettico poiché credeva che il reclamo del La Marra sarebbe stato inutile, in quanto l'Inghilterra si riteneva in diritto di predare le navi napoletane in Oceano (Tanucci, 1985: 852-853 e 876), fatto che si verificò ben presto. Il I5 settembre dello stesso anno Tanucci scriveva al re che oltre la Vittoria era stata catturata anche un'altra nave del La Marra, l'Amalia, dalle fregate inglesi nei mari d'America, benchè tutto il carico appartenesse a negozianti napoletani, rimarcando che l'Albertini riteneva inutile reclamare presso la corte inglese: egli spiegava infatti «che la ragione è il luogo cioè l'America ove non è lecito commerciare e navigare ai sudditi del re colli francesi, perché la Maestà Sua non possiede alcuna terra in America, per la quale li sudditi vi potessero avere un commercio precedente alla guerra [...] [aggiungendo] Vostra Maestà intende più di quello che io dico, e non devo esprimere» (Mincuzzi, 1969: 9I-92). Intanto il La Marra era partito per Londra, dove arrivò il 15 novembre. ${ }^{20} \mathrm{Ma}$ il commercio con le Americhe continuava: in Napoli giungeva da Antigua un'altra nave, la Regina Amalia, che dopo lo scarico delle merci doveva essere venduta probabilmente al capitano Costantino Stinca. ${ }^{2 \mathrm{I}}$

Il La Marra arrivò a Londra il I5 novembre I76r e Tanucci il 24 avvertì Albertini dell'arrivo del mercante, «partito pieno di buone speranze»; ma ben sapeva che gli inglesi erano convinti di poter e dover impedire il «commercio nei mari d'America a tutti quelli

I7 ASN, Farnesiano, I523, 16/10/1760.

I8 ASN, Farnesiano, I520, ff. I9o ss., I6/Io/I760.

I9 ASN, Esteri i/r e 5/2/1762.

20 ASN, Esteri, $15 / \mathrm{II} / \mathrm{I} 76 \mathrm{I}$.

2I ASN, Esteri, II/I2/I76r. 
che in tempo di pace non [avrebbero potuto] avervi commercio», ragione per lui «non ... buona» (Tanucci, 1988: 322-323)

Il 28 novembre scriveva anche all'abate Ferdinando Galiani, suo buon amico, che era a Parigi, per comunicargli riferendo che secondo l'Albertini era «impossibile la recuperatione della nave del La Marra» (Tanucci, I988: 325-326). Ed ancora il primo dicembre scriveva all'Albertini manifestando tutto il suo disappunto e concludeva: «Siamo deboli e doviamo soffrire così barbara pirateria», riponendo le sue speranze nella fine della guerra (Tanucci, I988: 33I). L'8 dicembre Tanucci reiterò le preghiere all'Albertini di aiutare La Marra, anche se concludeva con amarezza: «Vedo disperata l'Amalia e poco sperata la Vittoria», le due navi del mercante (Tanucci, 1988: 450-45I). Infine alcuni mesi dopo, il to agosto I762 scrisse all'Albertini che al La Marra era stato consigliato di risparmiare il denaro per la causa in corso, ritenendo impossibile recuperare la nave; ed aveva espresso le stesse considerazioni nella missiva settimanale al re del ro agosto (Tanucci, 1988: 317-318).

Ma la situazione all'improvviso si risolse: il 22 marzo 1763 Tanucci, mostrando tutto il suo sconcerto, scrisse a Madrid al duca di Losada, Giuseppe Miranda Ponce de Leon: «vergognoso è stato per [Albertini] incaricato della reclamatione della S. Amalia di Lucio La Marra l'averla data a noi sempre per dispersa e impossibile ad averla e poi facilmente conseguita lo stesso [... L La Marra quando in persona andò a Londra [mostrando] che col il governo brittanico più ha potuto un mercante che il Re» (Tanucci, I997 II: 36-38). Ma il La Marra non si era arreso: dopo un anno di permanenza a Londra aveva ottenuto la restituzione della Santa Amalia. Perciò Tanucci, che non aveva molta fiducia nelle abilità diplomatiche di Albertini, gli aveva scritto che forse in quel caso gli inglesi avevano avuto riguardo per il giovane re Ferdinando. ${ }^{22}$

Comunque, data la situazione critica, nel porto di Napoli si prendevano misure adeguate: non si permetteva alle navi da guerra inglesi di «carenare al Salvatore», cioè fare riparazioni, dal momento che «vi era poca guarnigione»; e ciò acuiva il malumore del console inglese. ${ }^{23}$

Ma le spedizioni commerciali in America non si erano fermate: il capitano sorrentino Costantino Stinca aveva organizzato un altro viaggio con una nave comprata a Londra; perciò Tanucci il I3 luglio I762 scriveva all'Albertini di sperare che il capitano avesse «un buon viaggio e un ottimo profitto", in modo da incrementare quei traffici (Tanucci, 1990: 255). Ancora una volta le sue speranze furono deluse poiché egli dovè comunicare al re, il I2 ottobre 1762 , che la nave, carica di merci provenienti «dall'Oceano», era stata catturata da sciabecchi algerini (Tanucci, I990: 445-448).

Fino al 1762 molti capitani e armatori sorrentini continuarono ad allestire navi per il «commercio con l'Oceano»: lo stesso I2 ottobre Tanucci riferiva al re che i capitani, malgrado le catture, continuavano a commerciare in Oceano richiedendo le esenzioni consentite, cioè il quinto sui diritti di dogana (Tanucci, I990: 445-448). L'ir ottobre I759 fu concessa la riduzione del quinto al negoziante Gennaro Buono su merci provenienti da Londra o inglesi vendute a Napoli. ${ }^{24}$ Il 29 luglio 1762 fu data la stessa concessione al negoziante Nicola Romano per merci esportate in Oceano tranne che per l'olio caricato a Gallipoli, destinato a Napoli, ed il 23 settembre fu dato un simile privilegio al negoziante Antonio Maria Lauro per merci poste su una nave destinata al commercio in Oceano. ${ }^{25}$ A queste concessioni si aggiungeva anche il privilegio dell'armamento al prezzo pagato dalla Regia Corte:l'ır ottobre r759, vennero concesse 2 cantara di polvere al capitano Carlo

22 ASN, Esteri, f. 205, I0/II/1762

23 ASN, Farnesiano, I523, ff. I58 ss., 26/10/176r.

24 ASN, Farnesiano, 1517, f. 20, II/10/1759.

25 ASN, Farnesiano, I526, ff. 25 ss., $29 / 7 / 1762$ e ff. 248 ss., $23 / 9 / 1762$. 
Gattola sorrentino, concessione rinnovata il 23 ottobre dell'anno seguente. ${ }^{26} \mathrm{Il} 3$ giugno I762 fu concesso a Niccolò de Turris di poter acquistare per la sua polacca cannoni di ferro acquistati da un bastimento danese ${ }^{27}$ per armare le navi. Oltre il La Marra e i Marchettu erano molto attivi i mercanti Nicola Palomba e Gennaro Rossi, che commerciavano non solo in Levante ma anche con navi dirette in Oceano. Il 28 agosto 1760 , al negoziante Palomba fu ordinato di far fare alla sua nave, che tornava da «luoghi sospetti», la prescritta contumacia di 40 giorni nell'isola di Nisida. Inoltre fu dato incarico all'abate Galiani, a Parigi, di «fare i suoi uffici per la determinazione della nave de' negozianti Rossi e Palomba circa la presa del bastimento del Padron Gargiulo». ${ }^{28}$ I mercanti Palomba e Rossi il I9 novembre I76r avevano ottenuto il rinnovo della concessione del quinto per altri due anni per le due navi costruite in sostituzione di quella perduta, che doveva caricare merci a Cirella, in Calabria (di solito uva passa, che si esportava in abbondanza in Inghilterra); ed il I5 aprile I762 era stata loro confermata la concessione data nel I76r per la polacca che doveva compiere un viaggio in Oceano anche per merci caricate sulla polacca da scaricare in Napoli e consegnare a commercianti regnicoli (non per quelli forestieri). ${ }^{29}$ Inoltre i due mercanti avevano relazioni di affari con quelli esteri, tra cui gli inglesi, residenti a Napoli, con i quali spesso nascevano controversie, come quella sorta con i negozianti inglesi Wildman e Commorell per la quale il 25 marzo I762 al Magistrato di Commercio di era stato dato ordine di continuare ad indagare sui motivi della lite. $3^{30}$

Dai dati riportati da Luigi De Rosa in merito agli approdi e alle partenze nel porto di Napoli nel i76o si ricava un quadro dell'articolato commercio dei mercanti Palomba e Rossi che operavano sia singolarmente che in società: tra le 6 navi inglesi approdate una trasportava merci e pesce salato per il Rossi e una simile merce per il Palomba; tra le $\mathrm{I} 6$ olandesi una trasportava merci per il Palomba; ed anche tra le 16 danesi una trasportava sarde salate per il Palomba, che riceveva merci anche da una nave irlandese. Invece il Rossi riceveva cuoi e piombo da una nave russa proveniente da Pietroburgo (De Rosa, 1968: 344 ss.).

Ma i problemi della corsa si riverberavano sui commerci: difatti spesso le navi mercantili venivano predate dai corsari inglesi, per cui essi subivano notevoli perdite, causando notevoli danni alla Compagnia di Assicurazioni Marittime: difatti i6 settembre 1762 era stato inviato a Giovan Battista Maria Jannucci, presidente del Supremo Magistrato di Commercio, il conto delle spese fatte dal Rossi per la restituzione di un bastimento predato da un corsaro inglese il cui carico era stato assicurato con la Compagnia delle Assicurazioni Marittime, che doveva pagare il dovuto. ${ }^{31}$

Oltre ai corsari vi era anche il problema della poca perizia dei capitani che si

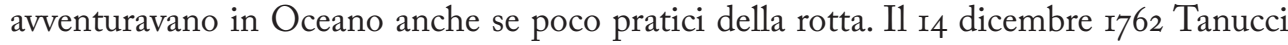
scriveva all'Albertini in merito al naufragio in Oceano della nave Ferdinando di Padron Canale commentando: «Vedo che siamo disgraziati sul mare. Veramente la marina è un'arte che deve ben studiarsi prima di azzardarsi a praticarla. Dio voglia che questi nostri [Marchetti, La Marra, Palomba, Rossi, ecc.] li quali avevano cominciato a farlo, e fino ora poco altro che mali, disgrazie, depredazioni han tirato dalla disciplina non siano dalla pace ricondotti all'inerzia» (Tanucci, 1990: 594). Perciò si cercava di istruire i piloti e

26 ASN, Farnesiano, I5I7, II/Io/I759, f. 20 e I520, 23/Io/I76o.

27 ASN, Farnesiano, I525, ff. 25I ss.,

28 ASN, Farnesiano, 1520, ff. 5I ss., 28/8/1760.

29 ASN, Farnesiano, I523, f. 6I ss., I9/II/I76I e I525, ff. II4 ss., I5/4/I762.

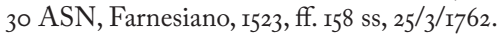

3I ASN, Farnesiano, I526, ff. 248 ss., I6/9/I762. 
timonieri delle galere con le lezioni di nautica del pilota Giuseppe Fileti, che oltre il suo stipendio percepiva 20 ducati per tale insegnamento. ${ }^{32}$

Uno dei problemi che Tanucci si trovò ad affrontare in quel periodo fu quello dell'accessione del Regno di Napoli al "patto di famiglia», il trattato concluso il i5 agosto I76r a Parigi fra Luigi XV di Francia e Carlo III di Spagna per unire più strettamente sul terreno politico-economico i diversi rami della famiglia borbonica e soprattutto le due monarchie di Francia e Spagna. Il Tanucci non vedeva di buon occhio questa alleanza per cui il 6 febbraio 1762 aveva scritto al principe di Cattolica, Giuseppe Bonanno e Filingieri, ambasciatore a Madrid (Schichilone, I979), con cui intratteneva una costante corrispondenza, che era conveniente per Napoli mantenere una completa neutralità. $\mathrm{Ma}$ Carlo III era di parere diverso e premeva sul ministro napoletano perché mutasse parere. Il Cattolica a sua volta cercava di persuadere il Tanucci che il trattato si poteva considerare una valida garanzia non solo per Francia e Spagna ma anche per il Regno di Napoli, facendo presente che Carlo si mostrava risentito. Di fronte a tanta insistenza il Tanucci sembrò cedere abilitando il Cattolica con dispaccio del I7 settembre $\mathrm{I} 762$ a sottoscrivere il patto: ma insisteva perché in una clausola fosse riconosciuto a Ferdinando il «diritto di alternativa» per i futuri trattati, non previsto nel testo. Il Cattolica si mise subito all'opera, elaborando un lungo memoriale che il 28 settembre inoltrò a Riccardo Wall, ministro degli esteri spagnolo, senza aver capito che l'inserimento della nuova clausola era un espediente escogitato dal Tanucci per ritardare la firma del patto, da lui avversato, perché temeva la irrequietezza della politica francese. La manovra ebbe successo: l'adesione al trattato fu rinviata e in seguito altri espedienti consentirono di ottenere lo stesso effetto fino a quando Carlo III non insistette più; così Tanucci riuscì ad evitare l'accessione al patto (Maiorini, 1991: 264-268).

Altro problema che impegnò il Caracciolo durante la sua missione diplomatica fu la cosiddetta «quistione delle visite». Conclusasi la guerra con l'Inghilterra (1763) i «fermieri» (controllori) dei porti di Provenza cominciarono ad attuare un controllo rigoroso dei bastimenti napoletani, facendo improvvise e minuziose ispezioni a bordo: ciò suscitò la pronta reazione del Tanucci che, anche su segreto suggerimento di Carlo, ordinò di applicare lo stesso trattamento per i bastimenti francesi che si trovavano nei porti del Regno o vi sarebbero arrivati. I diplomatici francesi protestarono vivamente e la corte di Parigi si appellò a Madrid dichiarando che «i fermieri di Provenza avevano agito all'insaputa del governo», denunciando i soprusi della reggenza napoletana. Tanucci rintuzzò prontamente le tesi di Parigi e il Caracciolo ne sostenne adeguatamente l'azione, insistendo perché Carlo ottenesse dal governo francese la instaurazione di un diritto di reciprocità in merito al controllo delle navi. Ma egli non riuscì a risolvere la questione, che ancora nel 1766 era motivo di reciproche lamentele (Scichilone, 1979).

L'arroganza degli inglesi, presenti con la flotta nel Mediterraneo dopo lo scoppio della guerra con la Spagna nel gennaio del 1762 , arrivò al culmine in febbraio, quando arrivarono a cannoneggiare Siracusa con 8 navi e fregate (Galasso, 2007-2008: 296-298). Questo continuo stato di incertezza della politica estera aveva creato forti preoccupazioni nel «re pupillo» e nei ministri; si temeva infatti un possibile attacco inglese con relativo bombardamento anche sulla Capitale. Perciò fu dato ordine al principe di Jaci, Stefano e Gravina, capitano generale delle forze di terra, membro del Consiglio di reggenza, di organizzare la costruzione di una serie di batterie in tutto il golfo con potenti cannoni in modo da difendere la Capitale e il Golfo. Perciò il 6 maggio I762 il re ordinava a Jaci di dare l'ultima mano alle batterie del Golfo ed il primo settembre dell'anno seguente

32 ASN, Farnesiano, 1538, Io/4/1766. 
furono stanziati 527 ducati per il «rivestimento di fabbrica» alla batteria posta alla punta di Posillipo, una delle più importanti, a difesa della Capitale. ${ }^{33} \mathrm{Ma}$, conclusa la pace poco tempo dopo, cessarono le ostilità: e forse proprio grazie a questo clima di distensione Tanucci scriveva al re il 19 marzo 1765 che il La Marra era riuscto ad avere ragione nella causa intentata nel foro napoletano contro gli assicuratori di Ancona che dovettero risarcirlo per i danni subiti dalle sue navi (Tanucci, I996b: 228-229).

In quel periodo molti padroni cominciarono a vendere le loro imbarcazioni a «forestieri amici», ottenendo il permesso dalla Corte, sempre che pagassero i diritti di esenzione ottenuti al tempo della costruzione delle loro imbarcazioni: il 3 febbraio ${ }_{7} 763$ fu concesso ad Antonio Longobardo di Castellammare di poter vendere la sua nave; stessa concessione fu data il I9 maggio a Michele di Costanzo, anche «fuori dei domini del re» ed il 25 settembre a Nicola de Turris di Castellammare fu concesso di vendere la sua polacca fuori del regno; il 29 dicembre fu concesso ad Antonio Maria Lauro di poter vendere due suoi pinchi «a nazionali o forestieri di nazioni amiche»; il I3 giugno 1765, fu permesso a Pietro Cafiero di Piano di poter vendere un suo bastimento ed il ro aprile I766, ad Antonio Cafiero di poter vendere la sua tartana. ${ }^{34}$ Questa tendenza può essere attribuita alla consapevolezza che senza lo stato di guerra erano di nuovo in auge le navi straniere. Ma si può anche supporre che i padroni di imbarcazioni costruite in Regno, specie nella costa sorrentina, avendo raggiunto una particolare abilità costruttiva riuscivano a trovare una clientela tra i padroni «forestieri». Perciò incrementavano le costruzioni utilizzando le franchigie previste dalla vigente legislazione per poi rivendere le imbarcazioni, entrando in un redditizio ciclo produttivo. Gli stessi mercanti Palomba e Rossi avevano diversificato il loro commercio, esportando «seta e lana in matassa» (Io.00o libbre il Palomba e 7.000 il Rossi) ${ }^{35}$ ed avevano venduto una loro polacca a Livorno. ${ }^{36}$

Si continuava perciò a dare attenzione alla flotta, in modo da proteggere le navi mercantili almeno in Mediterraneo: il 7 settembre del 1762 Tanucci scriveva al re che erano state mandate alcune galeotte a "protegger la fiera di Salerno»; invece le due fregate tornate da Ponente vi sarebbero tornate per proteggere i bastimenti mercantili che tornavano dai porti spagnoli, da Marsiglia, da Genova e da Livorno (Mincuzzi, 1969: 132).

L'anno seguente, l'in gennaio I763, Tanucci scriveva al re che su richiesta della Deputazione di Sicilia si era concesso di far restare «fissa in Sicilia una parte delle forze marittime del re anche perché nel paese restasse parte del denaro che la Sicilia paga[va] per l'armamento navale». Inoltre il Consiglio di Reggenza riteneva necessario fatto carico della necessità di «stabilire in Sicilia un arsenale», anche se con denaro derivante dai proventi della stessa isola (Mincuzzi, I969, I45-I46). Ma le galere e galeotte destinate alla corsa sulle coste meridionali della Sicilia dovevano andare a rifornirsi «di biscotto» nel porto di Agrigento, dove avrebbero fatto lo «spalmo» (carenaggio), non in quello di Palermo. ${ }^{37}$

Unadellepreoccupazioniprincipalieraperòquella diprovvedereall'approvvigionamento dei materiali di costruzione: nel I76o Tanucci aveva esaminato una nota in cui si elencava il numero e le dimensioni di alberi del Nord che occorrevano per la costruzione delle unità di grosse dimensioni in modo da dare ordine alla Corte di Spagna di organizzare

33 ASN, Farnesiano, 1525, ff. I8I ss., 6/5/1762.

34 ASN, Farnesiano, I528, ff. I6 ss., 3/2/1763; I529, ff. I6 ss., I9/5/1763; I530, ff. I57 ss., 25/9/1763; I531, ff. го9 ss., 29/I2/I763; I534, I3/6/I765; I538, I0/4/I766.

35 ASN, Farnesiano, I534, I3/6/I765.

36 ASN, Farnesiano, I53I, ff. I ss., I7/I/I763.

37 ASN, Farnesiano, I528, ff. I93t., 7/4/1763. 
questo acquisto..$^{8} \mathrm{Si}$ davano anche ordini per riparare la fregata San Carlo per il cui "controbordo» il 29 settembre 1762 si riferiva che erano stati spesi 800 ducati. ${ }^{39}$ Inoltre si approntavano due fregate, la stessa San Carlo ed un'altra da scegliere, per la nuova campagna..$^{\circ}$ Poi «si dispone[va] che il marchese Tanucci appur[asse] bene l'abilità del costruttore genovese Bartolomeo Bianchi» e sospendesse nel frattempo gli ordini dati per il taglio del legname occorrente per la nuova fregata; avrebbe dovuto dare ordine dopo un mese di far tagliare il legname occorrente in Sila: ${ }^{41}$ difatti il I9 aprile ${ }_{7} 633$ Tanucci scriveva: «Se Carlo [avesse] approva[to si sarebbe] costruito invece di una nuova galera una fregata di 32 cannoni [visto che] don Michele Reggio [aveva] fatto notar l'inutilità delle galere, dopo che gli algerini usa[va]no navi da guerra» (Mincuzzi, 1969: 155).

Dopo l'esame fatto fare da Tanucci da persone esperte, egli scriveva al re il I7 maggio I763 di aver fatto dare al Bianchi ordine di «fabricare il S. Filippo [...] fregata da 50 cannoni» per una spesa prevista di più di 8.000 ducati e il 2 I agosto lo informava della necessità di rifare la vecchia fregata S. Amalia (Mincuzzi, 1969: 157-158 e I70-I7I). Inoltre il 30 agosto riferiva che per il loro armamento su richiesta del generale Reggio erano stati acquistati «al prezzo vantaggiosissimo di 686 ducati io cannoni di ferro da 6» da una nave inglese di cui la marina aveva estremo bisogno (Mincuzzi, I969: I7I-I72, I32). Ma poiché ormai la fregata San Carlo era malandata e «non atta al corso» si ordinava di utilizzare al suo posto la fregata Concezione. ${ }^{42} \mathrm{Il} 27$ settembre ${ }^{2} 763$ Tanucci scriveva al re con rammarico che vi erano spese spesso inutili, come quella di molte migliaia di ducati sostenuta per riparare la fregata $S$. Carlo rivelatasi inutilizzabile, il che aveva suscitato le ire di Giovanni Asenzio Goyzueta, Segretario di Stato per la regia Azienda e per il Commercio dal 176r (Mincuzzi, I969: I74-I75). Si ricordava infatti che nel i76o per la fregata San Carlo erano stati spesi 7437.97 ducati e si prevedeva di spenderne altri 12.000 per cui si doveva decidere sul da farsi. ${ }^{43}$

In effetti la situazione del Regno era critica poiché il Mezzogiorno era stato prostrato da una terribile carestia, verificatasi nella prima metà del I764; e ad essa seguì una epidemia che falcidiò ulteriormente la popolazione. Tanucci si adoperò con ogni mezzo per acquistare grano da Trieste, con la mediazione della ditta Brentani, e da Marsiglia. Ed alcune quantità arrivarono anche dalla Spagna. Ma per lungo tempo il grano non bastò, per cui le costruzioni navali subirono un arresto.

Risolta la crisi granaria le costruzioni ripresero, per cui a fine anno fu varata la nuova fregata San Ferdinando. ${ }^{44}$ Poi Tanucci scrisse due lettere al re, il 20 e il 27 novembre I764, per comunicargli di aver ottenuto dal Papa la dispensa di poter costruire a Palermo due sciabecchi con la rendita della Badia di Partinico, che dovevano servire anche a difendere sia le coste adriatiche regnicole, pugliesi e abruzzesi, che quelle dello Stato della Chiesa, in primis l'importante porto di Ancona (Mincuzzi, I969: 243-244 e 244-245).

Qualche giorno dopo, il 4 dicembre, Tanucci riferì che l'intendente della marina aveva presentato una nota di spese molto alte ma necessarie per ripristinare le due fregate S. Amalia e Concezione e gli sciabecchi (Mincuzzi, I969: 245-246). Il primo gennaio dell'anno seguente Tanucci scriveva al cognato, il conte Giacinto Catanti, ministro plenipotenziario in Olanda, all'Aia, dal $\mathrm{I} 763$, della richiesta fatta dal generale Reggio di

38 ASN, Farnesiano, 1518, f. 134t., 22/3/1760.

39 ASN, Farnesiano, 1525 , ff. I59t. ss., $29 / 9 / 1762$.

40 ASN, Farnesiano, I528, ff.I ss., $2 / 1 / 1763$.

4I ASN, Farnesiano, I528, ff. II6 ss., Io/3/1763

42 ASN, Farnesiano, 1530, ff. 50 ss, $25 / 8 / 1763$.

43 ASN, Farnesiano, 1530 , ff. 59 ss., I/9/1763.

44 ASN, Farnesiano, ff. I64t., I/II/1764. 
acquistare alberi del Nord, del costo di 18.000 ducati, per i quali secondo il generale non era necessario «noleggiare un bastimento intero»; una settimana dopo gli riscriveva per sollecitarlo, visto che «vantaggiosissimo» acquisto aveva spinto il Reggio a chiedere al Tanucci che il cognato si adoperasse anche per l'acquisto di cannoni di ferro, da reperire in Svezia; inoltre il generale rimarcava che lo sciabecco S. Gabriello era «in malo stato», per cui si doveva ordinare il taglio di alberi in Calabria, anche se la zona era ormai «esausta» (Tanucci, I996: I9 ss. e 47-48). Perciò Tanucci lo stesso 8 gennaio aveva scritto al re che «spera[va] nei legnami di costruzione nuovamente scoperti nei luoghi farnesiani e medicei d'Abruzzo e in alcuni contigui della marchesa della Valle e del principe di S. Bono», Gregorio Caracciolo (Tanucci, I996: 48-52).

L'anno seguente, il ı766, si ebbe una netta ripresa delle costruzioni. Gli sciabecchi a Palermo erano quasi completati per cui il to aprile fu mandato il mastro delle vele a Palermo per la cucitura di quelle occorrenti alle due imbarcazioni e fu dato loro il nome rispettivamente di Santa Maria di Altomonte e e quello della vergine a cui era intitolata la Badia di Partinico. ${ }^{45}$ Lo stesso mese si provvide a fare il taglio di legname nella Sila di Cosenza per la costruzione della nuova nave a 60 cannoni, per il quale furono pagati 8II2 ducati. Altro legname fu tagliato nei boschi di Rosarno per la costruzione di sciabecchi, legname preso nei feudi del duca di Monteleone, che produsse un ricorso; altri 5 alberi per le galere giunsero da Cartagena e furono pagati al prezzo di quelli giunti dall'Olanda. Infine si ordinò di impostare altri due sciabecchi appena fosse terminata la costruzione a Palermo dei primi due. ${ }^{46}$ Pochi giorni dopo si dettero gli ordini per l'armamento della nuova fregata, in modo da procurare i cannoni necessari e furono pagati 1417.87 ducati per i lavori necessari per l'artiglieria. ${ }^{47}$ Altri 1700 ducati furono spesi per calafatare le tre fregate San Ferdinando, Sant'Amalia e La Concezione..$^{8}$ Ma viste le spese esorbitanti (15.53I,75 per i vari accomodi, 3.255 ducati per la «sartia» e 3.458 per ulteriore legname occorrente per la nuova nave a 60 cannoni) il re aveva richiesto all'intendente di fare ogni 6 mesi una relazione dettagliata di tutte le spese necessarie, che apparivano eccessive. Comunque si ordinava anche di fare la prova dei 24 cannoni acquistati per gli sciabecchi, per vedere se erano adatti e di buona qualità, in modo da pagare il negoziante inglese, se l'esito fosse stato positivo e chiedergli di procurare altri cannoni. ${ }^{49}$ Inoltre Tanucci scrisse al console di Trieste di mandargli notizie sulla possibilità di acquistare cannoni, visto che i cannoni di ferro rimasti erano inutilizzabili..$^{\circ}$

Comunque gli sciabecchi e l'«Armata sottile» di galere e galeotte continuavano ad esercitare il «corso» per proteggere le navi mercantili napoletane in Mediterraneo. Il Tanucci il 18 marzo I766 riferiva che secondo gli ordini del generale Reggio: «due sciabecchi [dovevano] croci[are] le acque tra Brindisi e Taranto, due tra Messina e Crotone; due con una galeotta la costa meridionale di Sicilia; due galeotte e una galeottiglia l'Adriatico; due galere li mari di Toscana; finalmente una galera e una galeotta [dovevano rimanere] in Napoli per quel che [poteva] occorrere; le due galere si volevano mandare al solito anzi tre nella costa meridionale di Sicilia, ma [Tanucci disse al re che sarebbero state] con la solita confidenza la primavera, l'estate e l'autunno nei porti di Palermo e altri della Sicilia senza uscir mai al mare, come avevan fatto in tutto il tempo della reggenza». Perciò fu dato ordine al viceré di far riattare il porto di Palermo, «molto ripieno e per la

45 ASN, Farnesiano, 1538 , Io/4/1766

46 ASN, Farnesiano, 1538 , 10/4/1766

47 ASN, Farnesiano, 1538, 24/4/1766

48 ASN, Farnesiano, $1538,8 / 5 / 1766$.

49 ASN, Farnesiano, $1538,15 / 8 / 1766$.

5o ASN, Farnesiano, 1538, 3/9/1766. 
maggior parte senza fondo» per la negligenza del Senato che aveva «lasciato perdere il pontone destinato a mantenere pulito il porto» (Mincuzzi, 1969: 308-309). In particolare due sciabecchi il San Ferdinando e il San Pasquale, dovevano fare il corso lungo le coste della Calabria, un luogo esposto e soggetto agli assalti nemici. ${ }^{5^{2}}$

Nello stesso tempo continuava il lavoro di ripristino dei principali porti iniziato in epoca carolina: si dava attenzione ai lazzaretti, necessari per lo «spurgo» delle merci provenienti dal Levante, dove la peste era endemica. Furono perciò dati ordini per il ripristino dei lazzaretti di Messina e Brindisi, unici porti in cui era possibile far entrare le armate..$^{52}$ La maggiore attenzione fu data a lazzaretto di Nisida, dove approdavano le navi provenienti dal Levante che poi dovevano scaricare le merci nel porto della Capitale: qui ne 1765 fu deciso di porre delle colonne di pietra a mo' di bitte e «fare un braccio di porto» con una stanza, il primo per l'approdo delle navi e la seconda per i funzionari della Deputazione della Salute che dovevano controllare gli approdi e le quarantene, lavori commissionati all'ingegnere Giovanni Bompiede, capitano del porto di Napoli, che doveva fare una accurata relazione dei costi. ${ }^{53}$ Poi l'anno seguente fu iniziata la costruzione, a cui contribuì la città di Napoli con 2.500 ducati, sempre sotto la supervisione dell'ingegnere Bompiede. ${ }^{54}$ Così fu fatto un totale ripristino del porto e del lazzaretto (Buccaro, 1993: I38 ss.).

Invece non fu accolta la richiesta di rifare ex novo il lazzaretto di Manfredonia, porto importante per i collegamenti con Venezia e Trieste, in quanto si riteneva che i 6.000 ducati raccolti dalle «abolite franchigie degli ecclesiastici» potevano bastare solo per il riattamento del vecchio. ${ }^{55} \mathrm{Si}$ continuarono anche i lavori iniziati nei principali porti in epoca carolina e nel porto di Crotone fu posto un argano per «carenare» gli sciabecchi che facevano il «corso» tra il mar Ionio e l'Adriatico. ${ }^{56}$

Data l'incertezza della situazione politica ed il timore di un assalto al Regno e soprattutto alla sua Capitale, che poteva essere bombardata se non si fossero prese le dovute precauzioni, si decise di fare una revisione generale delle batterie poste in tutto il Golfo napoletano e dell'artiglieria che vi era stata montata: così fu stilata una precisa relazione, in modo da tenere sotto controllo i luoghi più importanti per la difesa: i punti più nevralgici erano il molo con i suoi magazzini, la fonderia, i mulini, il Castel nuovo, i castelli del Carmine, di Sant'Elmo e dell'Ovo, la punta di Posillipo, l'accademia di Marina ed il porto del Granatello, prospiciente la reggia di Portici, usato dal re per pescare.

Tabella I

«Relacion de la Artilleria de Bronze regular y de Nuevo Methodo ... en los Parages...»7

\begin{tabular}{|l|c|l|l|c|l|}
\hline \multicolumn{1}{|c|}{ Luogo } & $\begin{array}{c}\mathrm{N}^{\circ} \\
\text { cannoni }\end{array}$ & \multicolumn{1}{c|}{ Tipo } & \multicolumn{1}{c|}{ Luogo } & \multicolumn{1}{c|}{$\begin{array}{c}\mathrm{N}^{\circ} \\
\text { cannoni }\end{array}$} & \multicolumn{1}{c|}{ Tipo } \\
\hline Castel dell'Ovo & 20 & Regolari da 24 & Magazzini & 2 & Nuovo met, da I2 \\
\hline Sermoneta & $\mathrm{I}$ & Regolari da 24 & Fonderia & 8 & Nuovo met, da I2 \\
\hline Magazzini & 6 & Regolari da 24 & Magazzini & 30 & Nuovo met, da 6 \\
\hline Carmine & 2 & Regolari da 24 & Accademia & I & Nuovo met, da 4 \\
\hline
\end{tabular}

5I ASN, Farnesiano, 1538, 24/4/1766.

52 ASN, Farnesiano, I523, ff. IoIt. ss., 3/12/176r e I525, ff. II4 ss, I5/4/1762, Messina; I522, ff. 33 ss., 8/4/176r, Brindisi.

53 ASN, Farnesiano, 1530, ff. 157t. ss, 25/9/1763; 1534, 27/6/1765, 8/8/1765; 1536, 27/2/1 766 .

54 ASN, Farnesiano, 1538, 24/4, I5/5 e 22/5/1766; 1537, 7/8/1766.

55 ASN, Farnesiano, $1525,26 / 9 / 1765$.

56 ASN, Farnesiano, 1523 , ff. 158 ss., $24 / 12 / 176 \mathrm{I}$.

57 ASN, Casa Reale Antica, 870, II/3/1766. 


\begin{tabular}{|l|c|l|l|c|l|}
\hline Molo & 28 & Regolari da 24 & Fonderia & 45 & Nuovo met, da 4 \\
\hline Gaeta & 2I & Regolari da 24 & & Mortai & \\
\hline Granatello & 4 & Regolari da 24 & Castel Nuovo & 4 & Da I3 pulg \\
\hline Mulini & 2 & Regolari da I6 & Molo & 2 & Da I3 pulg. \\
\hline Granatello & 4 & Regolari da I6 & Castel Ovo & 4 & Da I2 pulg. \\
\hline Carmine & 6 & Regolari da I6 & Carmine & 2 & Da I2 pulg. \\
\hline Molo & 6 & Regolari da I6 & Gaeta & I6 & Da I2 pulg. \\
\hline Gaeta & 33 & Regolari da I6 & Posillipo & 2 & Da 9 pulg. \\
\hline S.Elmo & 9 & Regolari da I6 & Magazzini & I & Da 9 pulg \\
\hline Magazzini & 8 & Regolari da 8 & Mulini & & Da 9 pulg \\
\hline Accademia & I & Regolari da 4 & Gaeta & & Da 9 pulg \\
\hline Magazzini & 4 & Regolari da 4 & S.Elmo & & Da 9 pulg \\
\hline Posillipo & 2 & Nuovo metodo da 24 & & Obici & \\
\hline Pietra Nera & 4 & Nuovo metodo da 24 & Fonderia & & Da 8 pulg \\
\hline Fonderia & I2 & Nuovo metodo da 24 & & & \\
\hline
\end{tabular}

Raggiunta la maggiore età, il I2 gennaio I767, al compimento del sedicesimo anno, il re iniziò il suo governo, sia pure coadiuvato dai ministri della abolita Reggenza. In un primo tempo il ministro Tanucci aveva dichiarato di voler rallentare la sua attività, data la sua cattiva salute. Ma i problemi degli anni precedenti, in primis le contese per l'accessione al Patto di Famiglia e le cause per i grani acquistati a Trieste e Marsiglia durante la carestia, non erano stati risolti. Perciò Tanucci, probabilmente su richiesta di Carlo, restò al fianco del giovane Ferdinando, ancora bisognoso di una guida sicura (Maiorini, r99r: 483 ss.).

\section{Il REgNo di FERdinANDO}

Un primo momento di crisi al termine della Reggenza si ebbe quando fu decretata l'espulsione dei Gesuiti dal Regno, sulla scia di quanto fatto dalla monarchia spagnola. L'espulsione era stata decisa da Carlo e Tanucci fin dall'inizio del 1767 ma solo il 3 novembre, dopo reiterati ordini la marina «si [era] finalmente mossa» e si [era] dato l'avvio di quanto disposto per la loro espulsione (Mincuzzi, I969: 4II-4I2). Tanucci il Io novembre avvertì Carlo che il giorno dopo sarebbe stata stampata la prammatica e sarebbe cominciato l'imbarco sulle navi dei gesuiti, mentre venivano sequestrati tutti i beni (Mincuzzi, I969: 4I2-4I3); ed il primo dicembre riferì al re che ne erano stati espulsi già 240 (Mincuzzi, I969: 415-416). Tra i beni sequestrati ai gesuiti vi erano i collegi in Napoli e nei capoluoghi delle province, riaperti secondo la normativa prevista fin dal I767 da Antonio Genovesi, perfezionata dopo la sua morte da Giacinto Dragonetti. Nel I770 furono istituiti i collegi per pilotini di San Giuseppiello a Chiaia, in Napoli, e di Meta e Carotto nella penisola sorrentina, creati per riorganizzare in modo capillare l'istruzione nautica (Sirago, 20I2b e 20I9).

Altri problemi si verificarono per il Tanucci dopo il matrimonio del re con Maria Carolina, figlia di Maria Teresa d'Austria, celebrato per procura il 7 aprile 1768 , con il quale si creavano nuovi equilibri che preoccupavano il ministro (De Lorenzo, 2008). Per il matrimonio fu spesa una somma enorme che prosciugò le casse statali, aumentando le preoccupazioni del Tanucci che manifestava la sua preoccupazione a Carlo (Ferrari, 2003: XXv ss). 
Il problema del ripristino della flotta era però ancora irrisolto, sia per la mancanza del legname che per la poca perizia dei costruttori: difatti il 2 ottobre I770 Tanucci riferiva al re che il vascello da guerra San Giuseppe «costrutto di fresco» era già ridotto in cattivo stato e il re aveva dovuto approvare «la grave spesa per metterlo in stato di servire» (Mincuzzi, I969: 636-638). Bisognava poi completare quanto previsto da Carlo alla sua partenza per mettere in sicurezza il Regno. Uno degli interlocutori privilegiati era il Cattolica: rientrato in Napoli il 22 settembre 1770 per ricoprire il prestigioso incarico di cavallerizzo maggiore della regina, come richiesto da Carlo, che lo voleva premiare per i suoi buoni uffici nel decennio in cui era rimasto in Spagna, assunse una posizione di spicco a corte (Scichilone, 1979). Ciò gli permise di collaborare strettamente col Tanucci, a cui riferiva quanto si diceva nell'entourage di Maria Carolina. Il Cattolica, al contrario di Tanucci, era favorevole a incominciare a mettere al corrente, sia pure in privato, la regina dei più importanti problemi politici, facendosi portavoce del desiderio della regina di occuparsi degli affari di stato: infatti Tanucci il I7 ottobre 1769 aveva scritto al re «apparisce un gran desiderio [della regina] d'entrar negli affari» (Mincuzzi, r969: $55^{8}$ ss.). In effetti secondo i capitoli matrimoniali la sua partecipazione doveva diventare ufficiale non appena avesse partorito il primo figlio maschio (Mafrici, 20Io: 49-80); ma ella già si apprestava ad inserirsi negli affari di governo.

In quei frangenti si temeva che la guerra con l'Inghilterra fosse inevitabile: ciò era avvalorato dalle lettere del marchese Domenico Caracciolo, inviato straordinario a Londra dal I764 in sostituzione dell'Albertini. Perciò Tanucci il 9 ottobre 1770 nella missiva settimanale aveva fatto presente a Carlo che la Francia offriva alla Svezia sussidi che invece avrebbe potuto dare al re: in tal modo si sarebbe potuta «qui pianta[re] una forza marittima di i2 vascelli da guerra coi quali il re, avendo preso il gusto della marina, forse s'indurrebbe a deporre la difficoltà delle bombe repentine sulla Capitale». Cattolica gli «aveva risposto che per quanto egli aveva potuto congetturare potrebbero facilmente li francesi annuire e anche alla Spagna potrebbe convenire una tal forza delle Sicilie sul mare». Perciò Tanucci ripropose il suo pensiero al re il quale «rispose che potendo mantener dodici vascelli da guerra [era] pronto a deporre ogni inquietudine». Infine Tanucci ottenne dal re di poter discutere di tale questione con Carlo, aggiungendo che secondo il suo parere sarebbe venuto molto «utile [...] a Lipari, Sorrento, Amalfi, Gaeta, Trapani, Bari, Procida Ischia di tale aumento della marina e quanto rispetto alla bandiera nel levante e nell'Africa, onde anche al commercio verrebbe un considerabile ajuto» (Mincuzzi, r969: 638- 639).

Poi il 20 novembre 1770 aveva scritto a Carlo che, mentre era a Procida col re per partecipare alla caccia, aveva discusso a lungo con Cattolica in merito al «patto di famiglia», che il sovrano non intendeva firmare (Mincuzzi, 1969: 644). Era una questione che si trascinava da anni: Ferdinando temeva che gli inglesi avrebbero bombardato la capitale mentre i francesi chiedevano di togliere la possibilità di commerciare agli inglesi in tempo di guerra.

Intanto si era verificato un incidente diplomatico fra la Spagna e l'Inghilterra in merito al possesso delle isole Malvinas, odiarne Falkland, nell'Atlantico Meridionale, il che aveva suscitato molta preoccupazione in Ferdinando. Nel 1770 si era avuto uno scontro tra Regno Unito e Spagna in merito a tale possessosso per cui si arrivò sull'orlo della guerra aperta tra Francia, Spagna e Gran Bretagna: ma il venir meno dell'aiuto francese costrinse la Spagna a recedere e a porgere scuse alla Gran Bretagna (Green, I906: 326). Naturalmente i venti di guerra potevano riverberarsi anche sul piccolo stato napoletano, per cui la vicenda era stata seguita con molta attenzione: perciò il 30 ottobre Tanucci aveva scritto nuovamente a Carlo palesandogli i timori di Ferdinando in merito 
all'Inghilterra, concludendo: «il re teme che Londra mediti qualche colpo o nell'America o nel Mediterraneo», poiché non era possibile che avesse armato 53 navi solo per «trattar con dignità e vantaggio l'affare di Malvinas con Vostra Maestà» (Mincuzzi, 1969: 642643). Un modo per ribadire le posizioni del regno meridionale era secondo Tanucci quello di proibire le necessarie importazioni di baccalà, cosa però quasi «impossibile $[. .$.$] per$ l'uso universale che questi popoli ne fanno». Egli aveva riferito a Carlo il progetto e questi aveva discusso della questione con Cattolica che aveva risposto «di simili pesci che nel golfo del Messico si producono e sono di sapore, seccati che siano, migliori di quello del baccalà», concludendo «forse la Sicilia colli suoi Sali di Trapani potrebbe salar tanto pesce del quale abonda quel mare che supplisse al baccalà, che convenisse proibire» (Mincuzzi, I969: 642-643).

In realtà si voleva riorganizzare il commercio con l'Oceano come era avvenuto durante la «Guerra dei sette anni». Tanucci nel 1766 aveva fatto nominare il cognato Catanti ministro plenipotenziario a Copenhagen, in modo da ampliare il raggio di interesse verso il Mar del Nord, il Mar Baltico e le Americhe (Maiorini, 2000: I89 ss.). La corte danese era utile per l'acquisto di merci per la flotta, alberi del Nord e cannoni, da reperire in Svezia; ma era ancor più utile per il commercio nelle Americhe. Difatti le isole danesi di America San Juan e San Thomas, dette «Indie occidentali danesi», dal $\mathrm{I}_{752}$ possesso della monarchia, erano state dichiarate porti franchi (Dookhan, 1974). Perciò vi potevano commerciare anche i napoletani, in base al trattato di commercio con la Danimarca. Così il La Marra, pensando di intraprendere un proficuo commercio, riprendendo le antiche rotte, aveva inviato al Catanti una lista di generi che avrebbe potuto esportare. $\mathrm{Ma}$ il conte manifestava a Tanucci le sue perplessità sul commercio nelle Americhe poiché nei possessi danesi non c'erano case commerciali napoletane, per cui per il ritorno bisognava far preparare un carico a credito, il che non era molto semplice. $\mathrm{E}$ altre perplessità le esprimeva in merito al dazio imposto dalla Danimarca sui vini napoletani, il doppio di quello pagato per quelli spagnoli e portoghesi, perché considerati liquori. Perciò il profitto non sarebbe andato ai bastimenti napoletani, che difficilmente navigavano fino al Mar del Nord e al Mar Baltico, ma ai bastimenti danesi che acquistavano i nostri vini..$^{8}$

Ma le maggiori preoccupazioni erano per la difesa del Regno: si decise di incaricare il Catanti di acquistare 80 cannoni di ferro di calibro da 24 e $18 . .^{59}$ Inoltre era stata fatta una convenzione col negoziante inglese Carlos Curtler per acquistare altri 50 cannoni di ferro, di cui 30 di calibro a 24 e 50 a 18 per l'artiglieria di terra da porre sulle batterie del litorale per un costo di 18.000 ducati; ma si riteneva che la soluzione migliore fosse di commissionarli in Svezia tramite il Catanti. Infatti quelli venuti dall'Inghilterra per la marina per i reali sciabecchi sarebbero stati pagati 7 ducati e $1 / 2$ al cantaro, sempre che alla consegna fossero di buona qualità. ${ }^{60} \mathrm{Il}$ Catanti si mise subito in moto, ${ }^{6 \mathrm{I}}$ chiedendo a Monsignor Springton, inviato di Svezia alla corte di Danimarca, di fornirgli notizie sui cannoni, sui prezzi e sulla spedizione: poi il I3 settembre 1768 inviò una relazione al cognato accludendovi quella dello Springton. ${ }^{62} \mathrm{Il}$ generale del Rio ordinava però a Catanti di soprassedere in merito ai I2 piccoli cannoni che gli erano stati promessi poiché anche se il prezzo era discreto secondo il parere del generale Reggio erano troppo corti per le esigenze della Marina. ${ }^{6}$ Perciò Tanucci il 28 marzo 1769 scriveva a Carlo che si era

58 ASN, Esteri, 26I, Catanti da Cophenagen a Tanucci, I9/ı//1767.

59 ASN, Esteri, 26I, 28/6/1768.

6o ASN, Esteri, 26I, 26/6/I768, lettera di del Rio.

6r ASN, Esteri, 26I, 23/8/1768, Catanti a Tanucci.

62 ASN, Esteri, 26I, I3/9/1768.

${ }_{63}$ ASN, Esteri, 26I, I3/12/1768, Tanucci a Catanti. 
deciso di provvedere in Regno, dando ordine di fondere cannoni e mortai e fabbricare le palle per rifornire le batterie, prestando attenzione a quella di Portici, «ad una batteria al palazzo pretorio della Torre del Greco che forz[asse] il nemico ad attaccare di fronte il fortino di Portici ove si d[oveva]no situare sei pezzi di trentatre e sei mortari di dodici pollici a galeotte, e truppa di terra», in modo da impedire ai nemici di posizionarsi «nella riva di Pietra Bianca [vicino] Portici per le batterie delle Tre torri» ( Mincuzzi, I969: pp. 513-514).

Vi era la reale preoccupazione che si potesse verificare un attacco al palazzo reale di Portici dove re Ferdinando si recava spesso per cacciare o dedicarsi alla pesca nelle «peschiere reali». Il giovane re aveva fatto venire a corte una cinquantina di abitanti di Lipari, esperti marinai e pescatori, che voleva far addestrare e tenere presso di sé come una sorta di guardia personale (Mincuzzi, I969: 553 ss.). Ma Tanucci il ı8 dicembre I770 scriveva a Carlo per manifestare tutta la sua preoccupazione in quanto essi avevano molta influenza sul re: difatti egli trascorreva molto tempo con loro, facendo insieme anche gli esercizi militari; ed aveva fatto fare per loro anche una uniforme (Mincuzzi, I969: 448-449). In un'altra lettera del I2 marzo I77 il ministro riferiva che essi erano rozzi ed incolti per cui temeva che distogliessero il re, che aveva un'indole dedita più ai piaceri che alle cure dello stato, dai suoi doveri. Invece Ferdinando aveva continuato a frequentarli, imparando da loro a manovrare le galeottiglie reali (Mincuzzi, 66o ss.): il Tanucci riferiva a Carlo il I3 ottobre 1772 che il re aveva arredato le galeottiglie in modo sontuoso, con vasellame d'argento pagato 3000 ducati tratti dalla cassa allodiale (o personale del re) (Mincuzzi, I969: 769-770). E con queste imbarcazioni, per le quali nel I773 aveva fatto costruire un porticciolo al Granatello, sulla costa prospiciente il palazzo reale (Visone, 2008: 229 ss.), si dedicava alacremente alla pesca (Sirago, 2018a: 80 ss.). Questa attività preoccupava Tanucci perché il re trascurava spesso le sue incombenze, come aveva già osservato per il passato nella sua lettera a Carlo del 15 agosto 1769 , quando aveva riferito «Per andare a pescare il re rimandò la settimana passata il Consiglio d'Azienda da giovedì a sabato» (Mincuzzi, 1969: 544 ss.). Ma comunque il ministro il 4 giugno I77I faceva presente a Carlo con piacere che con la esperienza acquisita dai marinai nel sovrano si era sviluppata una certa «inclinazione alla marina» (Mincuzzi, 1969: 673-674).

Per gli altri porti si continuava invece a provvedere solo per le spese di ordinaria amministrazione. Il problema più urgente era quello di garantire la difesa della Capitale. Così Tanucci il ı8 dicembre 1770 da Persano, dove aveva accompagnato il re a caccia, scriveva a Carlo di aver discusso col principe di Jaci, su richiesta del re, in merito alla «difesa delle piazze marittime, e particolarmente di Napoli [...] difficile [in cui] le batterie da piantarsi a quest'oggetto nel littorale [dovevano] esser di cannoni [in bronzo] da 24 [già reperiti ] in numero bastante dai castelli [o da] alcune batterie superflue e inutili»; e per giugno si sarebbero potuti avere alcuni nuovi cannoni «disegnati da Valiére per difesa di quella larga baja che rende difficile la difesa di Napoli» (Mincuzzi, I969: 648-649). Nel I772, il 9 giugno, Tanucci ringraziava Carlo che aveva offerto il suo aiuto, promettendo di mandare 25 cantari di rame americano, da pagarsi coi proventi della cassa allodiale che voleva sostenere «la spesa tutta delle batterie e di tutto quello straordinario [...] per la fortificazione di questo litorale tra Pozzuolo e Castellammare» (Mincuzzi, I969: 746-747). Un mese dopo, il I4 luglio, Tanucci riferiva che anche se i lavori venivano effettuati con rapidità rimaneva il problema della mancanza dei cannoni, non solo quelli di ferro che si sperava, invano, di poter fare nelle ferriere di Calabria ma anche quelli di bronzo, per i quali, se si fosse effettuato il lavoro proposto dal marchese di Valére, sarebbe stato necessario spendere almeno 300.000 ducati (Mincuzzi, 1969: 752). 
Il I7 aprile 1774 Tanucci riferiva a Carlo che si era deciso di far venire i cannoni dalla Svezia; inoltre il re aveva ordinato «la costruzione nelle ferriere di Calabria del numero copioso di palle proposto dal comandante dell'artiglieria colla proporzionata dimensione di cannoni di a 33" (Mincuzzi, I979: 890-891). Poi il 2 maggio riferì che l'ingegnere Bompiede aveva fatto una accurata relazione dei lavori alle batterie del litorale, per le quali erano stati spesi 18.000 ducati dalla cassa allodiale e ne erano stati stanziati altri 6000 dallo stesso fondo (Mincuzzi, I979: 961-962).

$\mathrm{Ma}$ anche la Sicilia e i luoghi di frontiera destavano preoccupazione: il 4 giugno I77I Tanucci riferiva a Carlo che in una seduta della Giunta militare «un poco calda» si era discusso sulle opere difensive progettate dal colonnello Luca Ricci per Palermo; inoltre erano insufficienti le fortificazioni delle isole Tremiti «ove l'abate comanda anche il militare e per l'Abate che è sempre il generale dell'Ordine [vi è] un suo sostituto il quale dispone anche della pubblica salute». La Giunta decise di dare «subito [...] mano alle opere approvate sul littorale di Napoli» per le quali si stanziarono 7000 ducati e che si provvedesse al ripristino del porto di Brindisi, continuamente interrito, vuotando "con puntoni [draghe] il canale ripieno». Infine Tanucci concludeva la lunga disamina sulla difesa del Regno osservando: «Linclinazione [...] per la marina [mostrata dal re gli sembrava] opportunissima per la situazione delle Sicilie, la buona marina delle quali potrebbe essere utile a tutta l'augustissima casa essendo marittima la forza del nemico naturale» (Mincuzzi, 1979: 673-674).

In effetti Tanucci il 2I gennaio 1772 manifestava a Carlo tutta la sua preoccupazione per le ingenti spese per la marina, un settore delicato da affidare a persona esperta dopo la recente morte del novantenne Michele Reggio, generale delle forze marittime: il ministro, che non era mai stato in sintonia col generale, ricordava che sotto la sua «protezione [...] si son fatti contrabbandi infiniti che furti ancora infiniti sono stati commessi non meno al re che ai privati». Perciò esprimeva al re «la [sua] umile opinione che ora sia il tempo di fare quella situazione che si reputi più conveniente per una materia tanto delicata» (Mincuzzi, 1969: 721-723).

Ma altri fronti di guerra si stavano aprendo: nel I768 era scoppiata la guerra russoturca per il dominio del Mar Nero, seguita con apprensione dal Ludolf, conclusasi nel I774 con la vittoria della Russia che aveva allargato notevolmente i suoi confini (Bottari, 20I8b). Perciò il I7 ottobre 1769 Tanucci aveva riferito a Carlo che re Ferdinando aveva ordinato di «non doversi ricevere nei porti delle Sicilie più di due navi russe per volta [...] di non dar loro provisione veruna e particolarmente da guerra e di far sapere alla Porta questi ordini che la Maestà Sua avrà dati in conformità dell'amicizia», che aveva ringraziato il Ludolf (Mincuzzi, I969: 558-560). In quegli anni molte navi e fregate russe dall'Acipelago greco erano arrivate nei mari del regno meridionale creando non poche preoccupazioni. Il 25 dicembre I770 Tanucci scriveva che «la zarina secondo il re voleva con questa guerra e pace consecutiva acquistar la privativa navigazione del mar nero per cui non accetta[va] alcuna mediazione e la pace [era] trattata solo dai visir e Romanow». Egli aggiungeva che anche il ministro Caracciolo nelle sue lettere da Londra riteneva «inevitabile la guerra e pensa[va] che il primo passo delle forze inglesi [sarebbe stato] nel Mediterraneo per cui [aveva] scritto alla Spagna di pensare a difendere la capitale» di Napoli, concludendo «io non vedo sicurezza e non mi fido ai nostri generali e molto meno agl'ingegneri, seppure abbiamo un ingegnere». A queste parole il re, si turbò ma tacque, mostrandosi d'accordo su quanto scritto dal Caracciolo, poiché se si voleva far rispettare la neutralità «bisogna[va] stare in armi e in difesa, e la capitale sicura da ogni insulto». Il colonnello Ricci aveva «cominciata la preparazione della fusa degli obus e di alcuni cannoni di bronzo", che sarebbero stati pronti in giugno. Intanto Caracciolo chiedeva 
istruzioni poiché riteneva questa guerra era diversa dalla passata, per cui le sue stesse mansioni erano più problematiche riguardo al ministro di Napoli in Londra. Secondo il Tanucci Carlo era «il primo attore mentre nell'altra entrò ad agire l'ultimo anno», per cui il ministro avrebbe dovuto essere supportato e avrebbe dovuto ricordare al re Giorgio III che Ferdinando non era nel Patto di famiglia, ovverosia il regno era neutrale» (Mincuzzi, I969: 650-65I).

In una lettera ancor più allarmata del primo gennaio I77I Tanucci scriveva a Carlo che aveva avuto notizie da Caracciolo sui movimenti della flotta inglese: era infatti stato «destinato il contrammiraglio How con ventiquattro navi da guerra al Mediterraneo» e si era diffusa la voce «di essersi risoluta una diversione delle forze di Spagna con attaccar le Sicilie e probabilmente le due capitali Napoli e Palermo, [...] le più facili ed in situazione la meno atta alla difesa». Al che re Ferdinando, in merito al «la necessità di premunirsi disse che «contra le venti e ventiquttro navi da guerra non [aveva] che opporre di equivalente» ma che avrebbe dato ordine di «provveder le piazza quanto si potesse [...] e che converrebbe unirsi il capitan generale col brigadiero Ricci per concertare il possibile e il necessario", anche se tra i due non correva buon sangue: perciò al Tanucci era affidato il compito di assistere ai colloqui tra i due per decidere il da farsi, data l'anzianità di Del Rio, che aveva « molto perduto colla gravissima età del suo nativo vigore». Invece la difesa di Palermo venne affidata al brigadiere Jauck. Questa situazione di incertezza preoccupava non poco Tanucci per i negativi risvolti sul commercio; egli osservava con lucidità: «Tra le mie ipocondrie della mente va che gl'inglesi forzato che abbiano con un insulto ingiustissimo il re ad accedere al Patto di famiglia, abbiano da accorgersi che mancherà loro in tempo di guerra tutta l'Italia, eccettuato Livorno, riguardo ai porti e al commercio loro dell'Italia stessa e del Levante onde abbiano a procurarsi con quanta forza potranno un ricovero come nella guerra grande della successione di Spagna si procurarono Gibilterra e Maone. Se mai pensassero questo dubito che Messina è in pericolo, ove non è un abile comandante, ove non si sono mai finite quelle fortificazioni che Vostra Maestà ordinò, ed ora ultimamente mi ha scritto confidenzialmente il viceré che mancano anche li viveri quotidiani». Infine manifestava la sua perplessità in merito all'ultima lettera del Caracciolo in cui si evinceva «che non sono gl'inglesi né preparati né disposti alla guerra, e sempre più celebra il re il decoroso contegno e disposizioni di Vostra Maestà per la guerra, la quale poche volte è stata e poche volte sarà probabilmente in avvenire tanto opportuna quanto lo è ora, per mansuefar la ferocia impossibile di questa nazione, la quale non ha ora alleati né denaro da provvedere nel continente, se tali non divengano li russi, che ora si trovano in queste parti con qualche truppa da sbarco» (Mincuzzi, 1969: 652-653).

Intanto i russi erano sempre più presenti nel Mediterraneo: il Tanucci il r9 maggio I772 riferiva a Carlo che il principe di Villafranca, Fabrizio Alliata Colonna, scriveva che in Messina vi erano «oltre la [...] nave russa con uno dei due generali, [Grigorij Grigor'evič] Orlow, [favorito della zarina] altri tre bastimenti armati russi in uno dei quali [vi era] un ambasciatore russo che [voleva] far la contumacia e quarantena in quel lazzaretto»; perciò il Villafranca aveva intimato loro di salpare, cosa che avrebbero fatto appena fossero "passati li venti» (Mincuzzi, 1969: 74I-742).

Tanucci il 5 febbraio I77I aveva scritto che per la difesa del regno in caso di guerra si cercava di riorganizzare l'armamento, anche se il colonnello Ricci riferiva che non riusciva a fare la fusione dell'artiglieria di ferro come promesso; lo stesso Jaci conveniva col Ricci che non vi era bastevole artiglieria di bronzo da 24 quanta ne occorreva per fornire le batterie necessarie per la difesa di Napoli dalle bombe e che mancava ogni genere di armamento, comprese le navi necessarie (Mincuzzi, I979: 658-659). Il I4 maggio 
dello stesso anno il ministro scriveva a Carlo che si sperava in artiglieria «da fabbricarsi in Francia per la difesa delle due capitali delle Sicilie architettata in parte dal marchese di Valiere proposta dal Ricci e dalla Giunta Militare e approvata» dal re. Per il Ricci occorrevano 80.000 ducati per l'artiglieria francese necessaria per la difesa delle due capitali; ma le casse statali erano vuote, anche per le esorbitanti spese di corte. Si era riusciti a raccogliere dalle rendite allodiali circa 30.000 ducati, ma Tanucci esortava il sovrano a non gravare su un popolo già povero, evitando di esigere altro (Mincuzzi, I969: 669-671).

Tanucci il 2I maggio ed il 9 luglio aveva avvertito Carlo che si continuava a provvedere alla difesa mentre si costruivano unità di combattimento come le galeotte, da sostituire alle vecchie, in modo da averne sei in totale (Mincuzzi, r969: 671-672 e 682-684). Inoltre il I7 settembre riferiva che il re «mosso dall'amore verso li suoi sudditi che di mare vivono mentre aderì a disarmarsi il vascello San Giuseppe volle che si mantenessero nell'inverno armate due fregate per protegger la navigazione di più di 200 piccoli e mediocri bastimenti [...] [che commerciavano tra le] isole del re e quelle di Francia» (Mincuzzi, r969: 697698). In un'altra lettera del I9 maggio $\mathrm{I} 772$ il ministro riferiva a Carlo che, secondo i suoi ordini, le galere non erano state abolite, anche se Ferdinando, divenuto esperto di imbarcazioni grazie ai liparoti, riteneva «che non rend[ev]ano le galere servizio che non si po[tesse] ugualmente dalle galeotte e sciabecchi». Difatti il numero delle galeotte era aumentato a sei come anche quello degli sciabecchi, in cui erano compresi i due costruiti a Palermo con le rendite della badia di Partinico (Mincuzzi, 1969: 741-742).

Tanucci il 20 agosto $177 \mathrm{I}$ riferiva a Carlo che il sovrano in un rapido ritorno della quiete alla corte di Francia visto che Carlo «ricomposte che sieno le cose di quella corte stima[va] trattabile la proposizione di aversi nelle Sicilie una forza marittima, che liber[asse] la Spagna e la Francia dal venirle a difendere dal nemico comune il quale non vi [sarebbe venuto] che per mare» (Mincuzzi, 1969: 69I-692). Ma il problema del reperimento dell'artiglieria era il più spinoso. Difatti Tanucci il 3I dicembre I77I scriveva che Caracciolo riteneva pericoloso richiederla in Inghilterra in quel particolare momento di crisi, per cui si doveva per forza ricorrere alla Francia, e per le necessità del Regno di buoni cannoni di bronzo si dovevano spendere almeno 300.000 ducati, somma di difficile reperimento (Mincuzzi, I969: 718-719). Il 25 febbraio 1772 ringraziava Carlo che approvava tale decisione e la spesa ben superiore ai 40.000 ducati previsti in prima istanza (Mincuzzi, I969: 727-729). Ancora il I7 marzo lo ringraziava per aver fatto acquistare il bronzo per l'artiglieria in Spagna, in modo da poter far risparmiare al Regno un ennesimo esborso (Mincuzzi, I969: 732-733). Il ministro poi il 9 giugno avvertiva Carlo dell'arrivo dalla Spagna di 25 cantari di rame americano da pagarsi con la cassa allodiale, in modo da completare la fortificazione del Golfo, da Pozzuoli a Castellammare, con le batterie (Mincuzzi, 1969: 746-747); ed il 29 settembre gli annunciava la costruzione di un nuovo sciabecco, sottolineandone la buona fattura: «Il nuovo sciabecco dotato di 32 cannoni è più fregata che sciabecco» (Mincuzzi, 1969: 766-767).

Ma la situazione rimaneva grave, per cui bisognava assolutamente riorganizzare il settore marittimo. Tanucci il 3 marzo 1772 riferiva a Carlo che Ferdinando aveva ordinato a don Antonio del Rio di cercare nella Segreteria di Guerra e Marina, di cui era presidente, il «piano pensato da [Carlo]d'istruzione marittima che [...] sapeva essere ottimo» (Mincuzzi, I969: 727-729). Visto che il piano era irreperibile, il ministro il I4 aprile riferiva a Carlo che era stato affidato ad una Giunta il compito «di formare le istruzioni marittime le più adatte alle circostanze e dello stato facendo l'uso opportuno delle istruzioni tali di Spagna e Francia e per simiglianza all'erario delle Sicilie delle danesi, svedesi e maltesi» (Mincuzzi, I969: 736-737). 
Carlo si era mostrato molto soddisfatto di questo «regolamento della marina» su cui si stava lavorando per limitare, secondo quanto emerso dalla relazione del capitano di galera Angosto, "gli errori le rubberie le frodi il danno enorme e continuo del real erario che si è sempre commesso nell'arsenale nelle costruzioni, negli armamenti da tutte le classi di quei che servono e han servito nella marina». Tanucci nella sua lettera del 26 maggio I772 ribadiva che tutto derivava dalla cattiva gestione del generale Michele Reggio che aveva protetto persone inaffidabili e corrotte, soprattutto il capitano delle maestranze (Mincuzzi, I969: 742-744). Intanto il colonnello Ricci era morto lasciando «li varj conti della fabbrica delle armi della Torre dalla Nunziata, dell'accademia militare, della fonderia dei cannoni e delle batterie per la difesa di questo litorale in molta confusione». Inoltre Antonio Del Rio, denunciava i «disordini grandissimi della marina, della quale era già il terzo anno che manca[va] l'intendente per l'apoplessia del povero Vicuña e per la mano che vi [aveva] preso Almagro capitano delle maestranze abusando della propensione del re per lui e profittando»; perciò Tanucci il 2 giugno scriveva a Carlo di averlo esortato a discuterne col re, valendosi «dell'occasione della Giunta nuovamente eretta della marina» (Mincuzzi, 1969: 744-745).

Comunque Tanucci il 24 novembre 1772 si complimentava con Carlo che era riuscito a persuadere il figlio «della inopportunità di dotare il Regno di altri I2 vascelli con il contributo di Spagna e Francia benchè il principe di Jaci [ritenesse] che solo così si [sarebbe potuto] mettere al sicuro Napoli e Palermo al sicuro dalle bombe inglesi» (Mincuzzi, I969: 777-779). Ma il i6 marzo I773 riferiva che si continuava a provvedere alla difesa: ai primi del 1773 vennero acquistati a Stoccolma con denaro «della cassa privata del re i8 cannoni del calibro di 24 e di 156 del calibro di 33", parte di un primo lotto, pagati 19.II5 ducati (Mincuzzi, 1969: 800). In totale vennero acquistati in Svezia I74 cannoni per ordine della Giunta delle fortificazioni; ma Tanucci esprimeva la sua perplessità rispetto al fatto che erano stati venduti 23 cannoni in ferro della Marina di differenti calibri e inservibili ma che secondo il ministro potevano essere utilizzati almeno negli attacchi di terra. Inoltre era stato venduto lo sciabecco vecchio San Fernando. Perciò Tanucci il 20 aprile 1773 , nel notare questa cattiva gestione, sottolineava che nei suoi sedici anni in Consiglio non aveva mai visto un bilancio di Marina (Mincuzzi, I969: 804-806). Ma le sue rimostranze non furono ascoltate: il 29 giugno scriveva a Carlo che erano stati acquistati in Svezia altri I2 cannoni di ferro da 8 e 24 da 6, pagati dalla cassa allodiale (Mincuzzi, 1969: 818-819); ed il 6 luglio aggiungeva che altri 10.000 ducati erano stati spesi per risistemare il vascello San Giuseppe e tre fregate (Mincuzzi, I969: 8I9-82I). Infine il 30 novembre riferiva che i 174 cannoni richiesti dovevano essere inviati nell'agosto dell'anno seguente (Mincuzzi, I969: 854-856).

Tanucci nella sua missiva settimanale del 26 mazo 1776 poteva concludere che la difesa del litorale, per la quale erano stati spesi con la cassa allodiale 30.000 ducati per le costruzioni e 40.000 per l'artiglieria venuta sia dalla Svezia che da Trieste, era completata, anche se ormai non sembrava così necessaria, visto che i venti di guerra si erano placati (Mincuzzi, 1969: I024-1025). Lo stesso giorno riferiva anche che il re prevedeva di spendere ro.60o ducati tratti dalla stessa cassa per il nuovo quartiere che egli voleva costruire per i volontari di marina, cioè i marinai di Lipari (Mincuzzi, 1969: 1022I024). Infine il 27 agosto avvertiva che su richiesta del Senato di Palermo si era pensato di ripristinare l'arsenale dove già erano stati costruiti gli sciabecchi con la rendita della Badia di Partinico, in modo da aumentare le costruzioni navali siciliane (Mincuzzi, I969: I049-I050). Ma questo fu uno degli ultimi atti di Tanucci. 


\section{Conclusioni}

Gli ultimi anni del ministro furono molto tristi. I rapporti con la regina divennero sempre più tesi perché ella mal sopportava il continuo colloquio e la gestione diretta, sia pure sotto forma di consigli, di Carlo. Tanucci aveva un ruolo delicato: riferiva a Carlo i comportamenti alquanto disdicevoli del figlio, dedito ai vari divertimenti, dalla caccia alla pesca al gioco d'azzardo, e poco incline ad occuparsi degli affari di Stato. Il ministro in una lettera del I8 giugno I77I aveva espresso la sua preoccupazione, chiedendo aiuto anche al confessore del re, a cui manifestava il desiderio di Carlo di esortarlo almeno a lasciare i giochi proibiti, dove perdeva molto denaro, a discapito delle casse statali (Mincuzzi, 1969: 677).

Intanto Maria Carolina, donna colta e attenta, si era inserita nel Consiglio di Stato ancor prima che nascesse il futuro erede, come riferiva Tanucci a Carlo il 9 luglio I77I (Mincuzzi, 1969: 682 ss.). In una lettera del 27 ottobre 1772 il ministro esprimeva tutto il suo scoraggiamento, ammettendo di essersi ormai «assuefatto alle irritazioni di Ferdinando e Maria Carolina per la quantità e continuazione delle quali [era] da molto tempo diventato la persona meno gradita e più aborita di tutta la Corte e condannato a servir chi non vorrebbe esserlo» (Mincuzzi, I969: 772-773). Il 27 luglio dell'anno seguente manifestava a Carlo tutta la sua preoccupazione per le spese eccessive in un momento tanto delicato e questi gli chiedeva di fare il possibile per limitarle (Mincuzzi, r969: 824). Il ro maggio I774 aveva scritto a Carlo mostrando apertamente la sua disapprovazione per le eccessive spese per le «feste carnevalesche», per cui era diventato oggetto di numerose mortificazioni (Mincuzzi, 1969: 888 ss.). Lo stesso era accaduto l'anno seguente, il 4 febbraio, quando aveva riferito a Carlo che per le stesse feste erano stati spesi 3.600 ducati (Mincuzzi, r969: 939).

Un altro scacco lo ebbe nella politica estera, dove si era sempre destreggiato abilmente. Dopo la fine della guerra russo-turca, nel I774, suggellata dalla pace di CüçüK Kaynarca (Bottari, 20I8b: 64 ss.) le corti madrilena e viennese fecero pressioni sulla corte napoletana per adottare una politica di apertura verso l'impero russo. Tanucci in una lettera a Carlo del primo agosto 1775 difendeva le sue idee conservatrici e i suoi pregiudizi nei confronti della Russia affermando che non solo i commerci con i nuovi mercati non erano necessari ma che essi avrebbero potuto favorire il contrabbando (Mincuzzi, I969: 986). Il mese seguente faceva notare che vi era un gran numero di navi inglesi e veneziane «che con Bandiera Russa passavano nel Mar Nero», non curandosi della mancanza di azione «del Corpo Diplomatico sull'affare della Libera Navigazione per le altre nazioni», ed esortava Ludolf a «non perdere di vista questo assunto». ${ }^{64}$

L'anno seguente continuò a raccomandare a Ludolf di gestire al meglio l'affare della navigazione del Mar Nero "che si dice[va] volersi stabilire», ${ }^{65}$ anche se il ministro credeva che la pace in Crimea non sarebbe stata durevole. Ma malgrado la strenua opposizione del Tanucci il governo napoletano cominciò ad intrattenere relazioni con la Russia tramite il marchese di Sambuca, Giuseppe Beccadelli Bologna, ambasciatore napoletano a Vienna (Barra, 1993: 99), Poco dopo la zarina inviò a Napoli il filosofo Frederich Melchior Grimm, suo «factotum», amico dell'abate Galiani, come inviato diplomatico (Scherer, I968). Tutto ciò aprì la via al trattato di commercio, fortemene voluto dal Galiani, firmato nel I787 (Sirago, 2018b).

64 ASN, ME, 7237/IX, Napoli, 2/o9/1775, Tanucci a Ludolf. 
A tutti questi dispiaceri si aggiungeva un perenne stato di cattiva salute, descritto minuziosamente al cognato Giacinto Catanti nelle sue lettere. Perciò non fa meraviglia se il 29 ottobre 1776 il Tanucci scriveva a Carlo un'ultima lettera in cui lo avvertiva di aver ricevuto un dispaccio nel quale gli si ordinava di consegnare tutte le sue carte al marchese della Sambuca, Giuseppe Beccadelli Bologna, nominato suo successore a sua insaputa (Mincuzzi, I969: Io66). Finiva così bruscamente una brillante carriera prima al seguito di Carlo, poi del figlio Ferdinando, cresciuto dal Tanucci con affetto paterno. In anni burrascosi egli era riuscito a conservare il trono per il re pupillo. Ma poi il cambio di rotta dato da Maria Carolina, che voleva togliere il regno dall'influenza spagnola per dirigerlo verso quella austriaca, fece ritenere inutile, anzi dannosa la presenza del Tanucci, che continuava a scrivere a Carlo ormai da privato cittadino, informandolo solo di avvenimenti di vita quotidiana (Borreca, 1976). Pochi anni dopo ella chiamò in regno l'ammiraglio di origine scozzese John Acton, provetto uomo di mare, a cui dette l'incarico di riorganizzare la flotta. E questi nel $\mathrm{I} 783$ fece costruire il regio arsenale di Castellammare nel quale furono varati 15 vascelli, fatti distruggere dal re a fine 1798 all'arrivo dei francesi prima di fuggire a Palermo (Formicola-Romano, 1990: 99 ss.).

\section{Bibliografia}

AJello, Raffaele (I976), «La vita politica napoletana sotto Carlo di Borbone. La fondazione e il tempo eroico della dinastia», in Storia di Napoli, II ed., Napoli, ESI, vol. IV, pp. 445-726.

_- (1977), «Carlo di Borbone re di Napoli e Sicilia», in Dizionario Biografico degli Italiani, vol. 20, www.treccani.it.

Anes, Gonzalo (I986), «Carlos III y Tanucci: influencias recíprocas durante los primeros años del reinado en España», in Raffaele Ajello e Mario D'Addio, Tanucci statista letterato giurista, Atti del Convegno Internazionale di studi per il secondo centenario $1783-1983$, Napoli, Jovene, pp. 59-8I.

Barra, Francesco (1993), Il Mezzogiorno e le potenze europee nell étà moderna, Milano, Sellino.

Broggia, Carlo Antonio (1979), Le risposte ai quesiti del console Balbiani, a cura di Antonio Allocati, Giannini, Napoli.

Bergoldt, Klaus (2004), «La fobia del contagio e le teorie mediche sul contagio», in Nelli Elena Vanzan Marchini (a cura di), Rotte mediterranee e baluardi di sanità, Venezia e i lazzaretti mediterranei, Ginevra - Milano, Skira, pp. I49-157.

Bianchini, Ludovico (197I), Storia delle finanze del Regno delle due Sicilie, Napoli, ESI, 2 voll.

Bono, Salvatore (2008), Un altro Mediterraneo. Una storia comune fra scontri e integrazione, Roma, Salerno.

Bono, Salvatore (20I6), Schiavi. Una storia mediterranea (XVI-XIX secolo), Bologna, Il Mulino.

Borreca, Luigi (a cura di) (1976), Il tramonto di Bernardo Tanucci nella corrispondenza con Carlo III di Spagna, ${ }_{776-1783}$, Palermo, Manfredi ed.

Bottari, Salvatore (20I4), «Trade and Politics between Naples, Sicily and The Ottoman Empire during the Kingdom of Charles of Bourbon (1734-1759)», in Halil Ersin Avci (a cura di), Türk Den;zc;lik Tar; $b_{i} B_{i} l l_{j} r_{i} l e r$, Proceedings of the International Symposium of Piri Reis and Turkish Maritime History (Prime Minister Ottoman) Archives in Istanbul, 26-29 settembre 20I3, Ankara, Türk Tarih Kurum, 20I4, pp. I49-155.

(2018a), Il Porto franco di Messina. Profili socio-economici e istituzionali, in Rosario Battaglia, Salvatore Bottari, Angela La Macchia, Porti e traffici nel Mediterraneo. Tre saggi di storia economica marittima (1695-186r), Milano, Franco Angeli, pp. 49-86. 
(2018b), Alle origini della Questione d'Oriente. Il conflitto russo-turco del I768-I774 e la diplomazia degli stati italiani, Biblioteca della «Nuova Rivista Storica», $\mathrm{n}^{\circ} 53$, Roma, Società Dante Alighieri.

Broggia, Carlo Antonio, Le risposte ai quesiti del console Balbiani (1979), a cura di Antonio Allocati, Napoli, Giannini.

BuccAro, Alfredo (I993), «I porti flegrei e l'alternativa allo scalo napoletano dal XVI al XIX secolo», in Giorgio Simoncini (a cura di), Sopra i porti di mare, II, Il Regno di Napoli, Firenze, Olschki ed., pp. I25-I54.

Carrino, Annastella (20I7), «Il Levante "que tenemos a la puerta". Progetti e pratiche del commercio mediterraneo nel Regno di Napoli di secondo Settecento», in Anna Maria Rao (a cura di), Napoli e il Mediterraneo nel Settecento Scambi, immagini, istituzioni, Bari, EDIPUGLIA, pp. 93-Iо7.

Cernigliaro, Aurelio (2012), B. Tanucci. Il Contributo italiano alla storia del Pensiero - Diritto, in www.treccani.it.

Clemente, Alida (2017), «Tra Europa e Mediterraneo: nuovi consumi e circuiti commerciali nella Napoli del Settecento", in Anna Maria Rao (a cura di), Napoli e il Mediterraneo nel Settecento Scambi, immagini, istituzioni, Bari, EDIPUGLIA, pp. 59-73.

D'Amora, Rosita (2003), «The diplomatic relations between naples and the ottoman empire in the mid-eighteenth century: cultural perceptions», in Oriente moderno, n. s., a. 22 (83), n. 3 , pp. 715-727.

De Lorenzo, Renata (2008), «Maria Carolina d'Asburgo Lorena, regina di Napoli e Sicilia», in Dizionario Biografico degli Italiani, vol. 70, www.trecccani.it

De Rinaldi, Giuseppe (2004), Napoli, Lazzaretto di Nisida, in Nelli Elena Vanzan Marchini (a cura di), Rotte mediterranee e baluardi di sanità, Venezia e i lazzaretti mediterranei, Ginevra - Milano, Skira, pp. 268-27I.

DE Rosa, Luigi (I968), «Navi, merci, nazionalità, itinerari in un porto dell'età preindustriale. Il porto di Napoli nel I760", in Studi sul Settecento Italiano, Napoli, Istituto Italiano per gli Studi Storici, pp. 332-370.

De Tiberiss, Giuseppe F. (200I), «Le "Riflessioni sopra, 1 commercio” di Federico Valignani. Alle origini del pensiero riformatore nel regno di Napoli», Frontiera d'Europa, anno viI (200I), n. I-2, pp. I65-280.

DiAz, Furio (I968), «L'abate Galiani consigliere di commercio estero del Regno di Napoli», Rivista Storica Italiana, $\mathrm{n}^{\circ}$ 80, pp. 854-909.

Dooknan, Isaac (1974), Danish Colonial Expansion. A History of the Virgin Islands of the United States, Kingston, Jamaica, Canoe Press.

Ferrari, Maria Claudia (2003), «Introduzione» a Bernardo Tanucci, Epistolario, xx, I768, a cura di Maria Claudia Ferrari, Napoli, Società Napoletana di Storia Patria.

Filesi, Teobaldo (I97I), «Un interessante capitolo delle relazioni tra Napoli e Tripoli: I739-I747», Clio, VI, n. 2, pp. 205-252.

Formicola Antonio e Claudio Romano (1990), «Il periodo borbonico (I734-I860)», in Arturo Fratta (a cura di), La fabbrica delle navi. Storia della cantieristica nel Mezzogiorno d'Italia, Napoli, ELECTA, pp. 6I-I56.

FortunAto, Nicola (I760), Riflessioni intorno al commercio antico e moderno del Regno di Napoli, Napoli, Stamperia Simoniana.

Füssel, Marian (2013), La guerra dei sette anni, Bologna, Società editrice il Mulino.

Galanti, Gennaro Maria (ig68), Della descrizione geografica e politica delle Sicilie, a cura di Franca Assante e Domenico Demarco, Napoli, ESI, , 2 voll.

Galasso, Giuseppe (2007-2008), Storia di Napoli, vol. 4, Il Mezzogiorno borbonico e napoleonico (I734-I8I5), UTET, Torino. 
(2018), «Genovesi: arretratezza e sviluppo del Mezzogiorno», in Anna Maria Rao (a cura di), Antonio Genovesi. Economia e morale, Napoli, Giannini, pp. 45-65.

Green, Walford Davis (1906), William Pitt, Earl of Chatham, and the Growth and Division of the British Empire, $1708-1778$, New York, G.P. Putnam's Sons.

Luise, Flavia (2016), «Reggio e Branciforte, Michele», in Dizionario Biografico degli Italiani, vol. 86, www.treccani.it

Mafrici, Mirella (2004), «Diplomazia e commercio tra il Regno di Napoli e la Sublime Porta: Guglielmo Maurizio Ludolf (1747-1789)», in Mirella Mafrici (a cura di), Rapporti diplomatici escambi commerciali nel Mediterraneo moderno, Soveria Mannelli, Rubbettino ed., pp. $15 \mathrm{I}-\mathrm{I} 72$.

- (2007), «Il Mezzogiorno d'Italia e il mare: problemi difensivi del Settecento», in Rossella Cancila (a cura di), Mediterraneo in armi (secc. xv-XvıII), Mediterranea. Ricerche storiche, 4 , 2 voll., II, pp. 637-663.

- (2010), «Un'austriaca alla corte napoletana: Maria Carolina d'Asburgo Lorena», in Mirella Mafrici (a cura di), All'ombra della Corte. Donne e potere nella Napoli borbonica I734I860, Napoli, Fridericiana editrice, pp. 49-80.

_ (2012a), «La diplomazia in azione nel Sette-Ottocento: rapporti commerciali tra la Russia e il Regno di Napoli», in Luigi Mascilli Migliorini e Mirella Mafrici (a cura di), Mediterraneo elè Mar Nero. Due mari tra età moderna e contemporanea, Napoli, ESI, pp. 3I-54.

— e Carmel Vassallo (a cura di) (2012b), Sguardi mediterranei tra Italia e Levante (XVI-XIX secolo), Malta, Università di Malta.

Maiorini, Maria Grazia (1985), «Introduzione» a Bernardo Tanucci, Epistolario, IX, I760-I76I, a cura di Maria Grazia Maiorini, Roma, Edizioni di Storia e Letteratura, pp. xxıII-xxıv.

_- (1991), La Reggenza borbonica (1759-1767), Napoli, Giannini ed.

- (2000), «Neapolitan diplomacy in the Eighteen Century policy and the diplomatic apparatus», in Daniela Frigo, Politics and diplomacy in early modern Italy. The Structure of Diplomatic Practice, 1450-1800, Cambridge University Press, pp. 176-209.

MARIN, Brigitte (2017), «Trasformazioni portuali a Napoli nel Settecento. La nuova strada della Marina e il paesaggio del litorale orientale», in Anna Maria Rao (a cura di), Napoli e il Mediterraneo nel Settecento Scambi, immagini, istituzioni, Bari, EDIPUGLIA, pp. 193-2II.

Mascilli Migliorini, Luigi e Mirella Mafrici (a cura di) (20I2), Mediterraneo elè Mar Nero. Due mari tra età moderna e contemporanea, Napoli, ESI.

Mincuzzi, Rosa (1969), Lettere di Bernardo Tanucci a Carlo III di Borbone (I759-I776), Roma, Istituto per la Storia del Risorgimento Italiano.

Musella Guida, Silvana (2016), «Relazioni politiche e commerciali tra il Regno di Napoli e la Porta Ottomana nei primi anni del regno di Carlo di Borbone. I doni per e da Mahmud I», in Annalisa Porzio (a cura di), Mondi Lontani Quaderni di Palazzo Reale, Napoli, pp. 9-28.

Pagano De Divitirs, Gigliola e Vincenzo Giura (1997), L'Italia nel secondo Settecento nelle relazioni segrete di William Hamilton, Horace Manne e John Murray, Napoli, ESI.

Passaro, Biagio (2019), «La navigazione mercantile napoletana nel Settecento e Ruolo e Consistenza della flotta mercantile napoletana nel xviı secolo», in Biagio Passero, Maria Sirago, e Pasquale Bruno Trizio (a cura di), Al servizio della Capitale e della Corte. La marineria napoletana nel Settecento, Napoli, ESI, pp. 5-34 e 35-62.

Pessolano, Maria Raffaela (1993), «Il porto di Napoli nei secc. XVI-XVIII), in Giorgio Simoncini ( a cura di), Sopra i porti di mare, II, Il Regno di Napoli, Firenze, Olschki, pp. 67-I23 e pp. Io7 ss.

Pezone, Maria Gabriella (2006), «Ingegneria idraulica in età borbonica: l'opera di Giovanni Bompiede», in Alfredo Buccaro, Gabriella Fabricatore e Lia Maria Papa (a cura di), Storia dell'Ingegneria, Atti del I ${ }^{\circ}$ Convegno nazionale, Napoli, 8-9 marzo 2006, Napoli, Cuzzolin ed., pp. 897-908. 
Pilati, Renata (200I), «Del commercio: G. Grimaldi e il riformismo napoletano nella prima età borbonica», Frontiere d'Europa, 7, nn. I-2, pp. 281-318 e trascrizione del manoscritto pp. 319-358.

Pirolo, Franca (20I7), «Peace treaties and trade between the Kingdom of Naples and Tripoli in the eighteenth century», Pedralbes, vol. 37, pp. I23-I34.

Reale Editto in cui si conferma alla città di Messina il privilegioi di Scalo e Porto (1785) Tradotto in lingua comune de' Greci per loro notizia e favore, Napoli, nella Stamperia Simoniana.

Rao, Anna Maria (2017), "Introduzione. Napoli nel Mediterraneo del Settecento", in Anna Maria Rao (a cura di), Napoli e il Mediterraneo nel Settecento Scambi, immagini, istituzioni, Bari, EDIPUGLIA, pp. 5-23.

Salzano, Achille (1924), La marina Borbonica, Napoli, Stab. Tip. Esperia.

Sbutega, Anton (20II), Il Montenegro tra Adriatico e Balcani, in Stefano Trinchese e Francesco Caccamo (a cura di), Rotte adriatiche. Tra Italia, Balcani e Mediterraneo, Milano, Franco Angeli, pp. 42-50.

Scherer, Edmond H. A. (ig68), Melchior Grimm, L'homme de letters, le factotum, le diplomate. Avec un appendice sur la correspondance secrète de Métra, Genève, Slatkine.

SchipA, Michelangelo (I923), Il Regno di Napoli al tempo di Carlo Borbone, Società Editrice Dante Alighieri, Roma.

Scichilone, Giuseppe (1979), «Cattolica Giuseppe Bonanno Filingieri principe di», in Dizionario Biografico degli Italiani, 22, www.treccani.it.

Setton, Kenneth M. (199I), Venice, Austria, and the Turks in the Seventeenth Century, Philadelphia, The American Philosophical Society.

SICKING, Louis (20I4), «Islands, pirates, privateers and the Ottoman empire in the early modern Mediterranean», in Dejanirah Couto, Feza Gunergun e Maria Pia Pedani (a cura di), Seapower, Tecnology and Trade Studies in Turkisk Maritime History, Piri Reis University Publications - Denizler Kitabevi, pp. 239-252.

Sirago, Maria (2004), Le città e il mare. Economia, politica portuale, identità culturale dei centri costieri del Mezzogiorno moderno, Napoli, ESI.

- (2008), «Città di mare e trasformazioni nell'assetto difensivo tra '700 e '800", in G. Amirante e M. R. Pessolano, Territorio, fortificazioni, città difese del Regno di napoli e della Capitale in età borbonica, Napoli, pp. 239-257.

(20I2a), «The Shipbuilding Industry and Trade Exchanges between the Kingdom of Two Sicilies and the Countries of the Baltic and the Black Sea (I734-I86I)», Mediterranean Review, vol. $5, \mathrm{n}^{\circ}$ 2, pp. 8I-IO7.

- (20I2b), «Gli studi nautici nel Regno di Napoli (I770-I86I)», in Mirella Mafrici e Carmel Vassallo (a cura di), Sguardi mediterranei tra Italia e Levante (XVII-XIX secolo), Malta, University Press, pp. 19-35.

- (20I2c), «Il consolato napoletano nel Mar Nero e lo sviluppo di Odessa tra la fine del Settecento e la prima metà dell'Ottocento», in Luigi Mascilli Migliorini e Mirella Mafrici (a cura di), Mediterraneo elè Mar Nero. Due mari tra età moderna e contemporanea, Napoli, ESI, pp. 203-233.

- (20I6), «La ricostruzione della flotta napoletana e il suo apporto alla difesa dei mari nel viceregno austriaco (I707-I734)», Archivio Storico per le Province Napoletane, pp. 7I-98.

- (2018), La flotta napoletana nel contesto mediterraneo (1503-I707), Ogliastro, Licosia ed. - (2018a), «Gli agricoltori del mare ascritti alla gleba», in Franca Pirolo (a cura di), La pesca in Campania e Sicilia. Aspetti storici, Ogliastro Cilento, 2 Licosia ed., pp. 2I-84.

- (20I8b), «Le rôle de Ferdinando Galiani à la signature du Traité de commerce entre la Russie et le Royaume des Deux-Siciles (1787)», in Tiran André Carnino Cecilia (diligence), 
- (2019), «L'istruzione nautica per la regia flotta e la marina mecantile del Regno di Napoli (I734-I799)», in Biagio Passero, Maria Sirago e Pasquale Bruno Trizio (a cura di), Al servizio della Capitale e della Corte. La marineria napoletana nel Settecento, ESI, Napoli, pp. 63-Iog.

Sola, Emilio (20I4), «The corsairs in the service of the Grand Turk: "Men of the frontier"», in Dejanirah Couto, Feza Gunergun e Maria Pia Pedani (a cura di), Seapower, Tecnology and Trade Studies in Turkisk Maritime History, Piri Reis University Publications - Denizler Kitabevi, pp. 253-263.

TAnucci Bernardo (I980a), Epistolario, I, I723-I746, a cura di Romano Carlo Coppini, Lamberto Del Bianco e Rolando Nieri, Roma, Edizioni di Storia e Letteratura.

-_- (I980b), Epistolario, II, I746-I752, a cura di Romano Carlo Coppini e Lamberti Nieri, Roma, Edizioni di Storia e Letteratura.

- (1984), Epistolario, IV, I756-I757, a cura di Lamberto Del Bianco, Roma, Edizioni di Storia e Letteratura.

- (1985), Epistolario, IX I760-I761, a cura di Maria Grazia Maiorini, Roma, Edizioni di Storia e Letteratura.

- (I988), Epistolario, x, I76I-I762, a cura di Maria Grazia Maiorini, Roma, Istituto Poligrafico e Zecca dello Stato,

- (1990), Epistolario, XI, I762-I763, a cura di Sergio Lollini, Roma, Edizioni di Storia e Letteratura.

— (1997), Epistolario, XII, I763-I764, a cura di Maria Claudia Ferrari, Napoli, Società di Storia Patria, 2 voll.

- (1996), Epistolario, XIV, I765, a cura di Maria Grazia Maiorini, Napoli, Società Napoletana di Storia Patria,

- (1996), Epistolario, Xv, I765, a cura di Maria Grazia Maiorini, Napoli, Società di Storia Patria.

- (2003), Epistolario, xx, I768, a cura di Maria Claudia Ferrari, Napoli, Società Napoletana di Storia Patria.

VArriale, Gennaro (20I7), «Conversioni all'ombra del Vesuvio (I565-I828)», in Anna Maria Rao (a cura di), Napoli e il Mediterraneo nel Settecento Scambi, immagini, istituzioni, Bari, EDIPUGLIA, pp. I43-I6o.

Venturi, Franco (i968), Settecento riformatore, vol I, Da Muratori a Beccaria, Torino, Einaudi.

VIsone, Massimo (2008), «La Villa d'Elboeuf e il Bagno della Regina al Granatello», in Maria Luisa Margiotta (a cura di), Il Real Sito di Portici, Napoli, paparo edizioni, pp. 225-240.

Wolf, John B. (1979), The Barbary Coast: Algeria under the Turks, New York, Norton.

ZAPperi, Roberto (1960), Aci Stefano, principe di, voce a cura di, Dizionario Biografico degli Italiani, I, I960, www.treccani.it

ZaugG, Roberto (2010), "Judging foreigners. Conflict strategies, consular interventions and institutional changes in Eighteenth-Century Naples», Journal of Modern Italian Studies, I3, 2, pp. I7I-I95. 